\title{
Multi time-step wavefront reconstruction for tomographic adaptive-optics systems
}

\author{
Yoshito H. Ono, ${ }^{1, *}$ Masayuki Akiyama, ${ }^{1}$ Shin Oya, ${ }^{2}$ Olivier Lardiére, ${ }^{4}$ David R. Andersen, ${ }^{4}$ \\ Carlos Correia, ${ }^{5}$ Kate Jackson, ${ }^{6}$ and Colin Bradley ${ }^{3}$ \\ ${ }^{1}$ Astronomical Institute, Tohoku University, 6-3 Aramaki, Aoba-ku, Sendai 980-8578, Japan \\ ${ }^{2}$ TMT-J Project Office, NAOJ, 2-21-1 Osawa, Mitaka, Tokyo 181-8588, Japan \\ ${ }^{3}$ Adaptive Optics Laboratory, University of Victoria, 3800 Finnerty Rd., Victoria V8P 5C2, British Columbia, Canada \\ ${ }^{4} \mathrm{NRC}$-Herzberg, 5071 West Saanich Rd., Victoria, British Columbia, Canada \\ ${ }^{5}$ Aix Marseille Université, CNRS, LAM (Laboratoire d'Astrophysique de Marseille) UMR 7326, 13388 Marseille, France \\ ${ }^{6}$ Division of Engineering and Applied Science, California Institute of Technology, 1200 E. California Boulevard, MC 155-44, Pasadena, \\ California 91125, USA \\ *Corresponding author: yo-2007@astr.tohoku.ac.jp
}

Received 17 November 2015; revised 20 January 2016; accepted 28 January 2016; posted 11 February 2016 (Doc. ID 253736); published 22 March 2016

\begin{abstract}
In tomographic adaptive-optics (AO) systems, errors due to tomographic wavefront reconstruction limit the performance and angular size of the scientific field of view $(\mathrm{FoV})$, where $\mathrm{AO}$ correction is effective. We propose a multi time-step tomographic wavefront reconstruction method to reduce the tomographic error by using measurements from both the current and previous time steps simultaneously. We further outline the method to feed the reconstructor with both wind speed and direction of each turbulence layer. An end-to-end numerical simulation, assuming a multi-object $\mathrm{AO}$ (MOAO) system on a $30 \mathrm{~m}$ aperture telescope, shows that the multi timestep reconstruction increases the Strehl ratio (SR) over a scientific FoV of $10 \mathrm{arc} \mathrm{min}$ in diameter by a factor of 1.5-1.8 when compared to the classical tomographic reconstructor, depending on the guide star asterism and with perfect knowledge of wind speeds and directions. We also evaluate the multi time-step reconstruction method and the wind estimation method on the RAVEN demonstrator under laboratory setting conditions. The wind speeds and directions at multiple atmospheric layers are measured successfully in the laboratory experiment by our wind estimation method with errors below $2 \mathrm{~ms}^{-1}$. With these wind estimates, the multi time-step reconstructor increases the SR value by a factor of $1.2-1.5$, which is consistent with a prediction from the end-to-end numerical simulation. (๑) 2016 Optical Society of America
\end{abstract}

OCIS codes: (010.1080) Active or adaptive optics; (010.1330) Atmospheric turbulence.

http://dx.doi.org/10.1364/JOSAA.33.000726

\section{INTRODUCTION}

Most large ground-based telescopes have single-conjugate adaptive optics (SCAO) systems [1-3], which correct phase distortion due to the atmospheric turbulence by using a wavefront sensor (WFS) and a deformable mirror (DM). These systems realize high-resolution observations in the near-infrared wavelengths. However, the correction of SCAO is effective only within a limited angle from a guide star (GS), known as the isoplanatic angle $\theta_{0}$. The limitation is caused by the optical path difference of a GS and a science target at high altitude in the atmosphere. The limitation is called angular anisoplanatism. The typical value of $\theta_{0}$ is roughly 10 arc sec in the $H$-band even at good observing sites [4].

Two wide-field AO (WFAO) concepts are proposed: multiconjugate $\mathrm{AO}$ (MCAO) [5] and multi-object $\mathrm{AO}$ (MOAO)
[6]. Both use tomography, which estimates the atmospheric volume to overcome the angular anisoplanatism and to enlarge the size of a scientific field of view $(\mathrm{FoV})$, where the $\mathrm{AO}$ correction is effective. These systems use multiple wavefront sensors aiming at GSs in different directions and observe the atmospheric turbulence above a telescope corresponding to the scientific FoV. Then, the three-dimensional structure of the phase distortion is estimated from WFS measurements by the tomographic wavefront reconstructor. Both systems use multiple DMs, but in different arrangements, to perform the correction. MCAO systems compensate for the phase distortion three-dimensionally by multiple $\mathrm{DMs}$, which are put in series and conjugated at different heights in the atmosphere. $\mathrm{MCAO}$ systems provide a uniform correction over a wide corrected $\mathrm{FoV}$, in which the $\mathrm{AO}$ correction is performed by DMs. 
In MOAO systems, there are multiple science pick-off arms on science targets in the wide scientific FoV. Each pick-off arm directs the light from one science target to a DM contained in each science channel. The DM applies the optimal correction to a small corrected $\mathrm{FoV}$ in its direction. This parallel approach increases the efficiency of the observation and can realize, for example, an observation of multiple galaxies with a multi-object integral field spectrogragh fed by MOAO corrected wavefronts [7-9]. MOAO systems require open-loop control because DMs correct the phase distortion in different directions from the WFS and this is challenging in terms of calibration.

The diameter corresponding to a given scientific $\mathrm{FoV}$ at different altitudes in the atmosphere is called the meta-pupil. It becomes larger at higher altitudes. As a result, there are areas covered by no or only one footprint of a GS optical path in the meta-pupils at high altitudes. The performance of the tomographic reconstruction is affected by these areas. In this paper, we refer to the area covered by no or only one footprint of a GS optical path as uncovered area or unoverlapped area, respectively. The uncovered area causes a significant tomographic error because there is no information from the WFS measurements in this region. The unoverlapped area also causes a significant tomographic error. The WFS probes a phase distortion integrated in the direction of its GS. In other words, a measurement of one WFS includes phase distortions at multiple altitudes, which causes degeneracy in the height direction. The lack of information on the uncovered and unoverlapped areas cannot be fully solved by the tomographic reconstructor leading to a large tomographic error even within the GS asterism.

In particular, the tomographic error due to the geometry of the GSs and the atmospheric turbulence becomes more severe when one considers the expanded scientific FoV of a WFAO system with a wide GS asterism. In addition, using a laser guide star (LGS) increases the geometric error compared with using a natural guide star (NGS) due to its conical optical path, which is called the cone effect. This means the geometric error will be more severe in WFAO systems for future extremely large telescopes (ELTs) [7-9], which have 25-40 m primary mirror diameters, with multiple LGSs because the cone effect worsens with aperture size.

The idea to use wind information for the WFAO control is studied for predictive control, which reduces the lag error resulting from the change of the atmospheric turbulence during the exposure time of the WFS and the computation time for AO corrections $[10,11]$. These predictive controllers allow the use of longer integration times of the WFS and/or real-time processing and therefore result in an increased limiting magnitude of GSs and improved sky coverage. In this paper, our method follows a parallel approach: it addresses reducing the tomographic error by increasing numerically the number of GSs, although it remains possible to expand it to include predictive control.

Our reconstruction method is based on Taylor's frozen flow hypothesis according to which the atmospheric turbulence layers move with a constant speed keeping its pattern of phase distortion. Under this assumption, we can estimate the time evolution of the measurements of the WFS at previous time-steps if we know wind speeds and directions, and the previous measurements can be used as the information for the tomographic reconstruction at the current time-step [12]. Our idea is to reduce the tomographic error due to the geometry of the GSs and the atmospheric turbulence by increasing the information using the measurements from both the current and previous time-steps simultaneously and thus to expand the corrected scientific FoV of WFAO systems.

Estimating wind speeds and directions at different altitudes is therefore essential. Several methods to estimate wind speeds and directions from measurements of Shack-Hartmann WFS (SH-WFS) are proposed and tested by using on-sky measurements $[13,14]$. These methods successfully detect the wind at multiple altitudes and start to be included in the real-time operation. We develop a wind profiler which estimates wind speeds and directions at each altitude by using temporal correlation of the phase distortion pattern reconstructed by the tomographic reconstruction.

The multi-step reconstructor is introduced and evaluated analytically in Section 2. In Section 3, we show its optimal performance based on an end-to-end (E2E) numerical simulation, assuming a MOAO system on a $30 \mathrm{~m}$ aperture telescope with multiple LGSs. The wind estimation method is outlined in Section 4. In Section 5, we present the performance of the wind estimation method and the tomographic reconstruction evaluated in the laboratory on RAVEN [15], a MOAO technical and science demonstrator installed and tested on the Subaru Telescope, and discuss a comparison between the laboratory test and a numerical simulation. Finally, we summarize our results in Section 6.

\section{TOMOGRAPHIC RECONSTRUCTION METHODS}

\section{A. Classical Single Time-Step Tomographic Reconstruction}

Here, we assume that the atmospheric turbulence consists of $N_{l}$ thin layers located at different altitudes. The aperture-plane phase of the light coming from a GS in the direction $\boldsymbol{\theta}=\left(\theta_{x}, \theta_{y}\right)$ at time $t$ is given by

$$
\boldsymbol{\varphi}(\boldsymbol{\theta}, t)=\sum_{i=1}^{N_{l}} \boldsymbol{P}_{\boldsymbol{\theta}}^{i} \boldsymbol{\phi}_{i}(t)=\boldsymbol{P}_{\boldsymbol{\theta}} \boldsymbol{\phi}(t),
$$

where $\boldsymbol{\varphi}$ is a column vector of distorted phase values on a discrete grid of points on the telescope aperture, $\boldsymbol{\phi}_{i}(t)$ is a column vector of a phase distortion on a discrete grid on the $i$ th turbulence layer at time $t, \boldsymbol{\phi}(t)=\left[\boldsymbol{\phi}_{1}^{T}(t) \cdots \boldsymbol{\phi}_{N_{l}}^{T}(t)\right]^{T}, \boldsymbol{P}_{\boldsymbol{\theta}}^{i}$ is a ray-tracing submatrix which extracts a phase distortion within a footprint of a GS optical path in the direction $\boldsymbol{\theta}$ on the $i$ th atmospheric turbulence by using a bilinear interpolation, and $\boldsymbol{P}_{\boldsymbol{\theta}}$ is a concatenation of all submatrices $\boldsymbol{P}_{\boldsymbol{\theta}}^{\boldsymbol{i}}$.

In MOAO systems, a tomographic reconstructor is determined to minimize the aperture-plane phase variance for each science direction $\boldsymbol{\theta}_{\boldsymbol{k}}$ [10],

$$
\boldsymbol{E}_{\boldsymbol{\theta}_{\boldsymbol{k}}}=\underset{\boldsymbol{E}_{\boldsymbol{\theta}_{\boldsymbol{k}}}}{\operatorname{argmin}}\left\langle\left\|\boldsymbol{\varphi}_{\boldsymbol{k}}-\hat{\boldsymbol{\varphi}}_{\boldsymbol{k}}\right\|^{2}\right\rangle,
$$

where $\boldsymbol{E}_{\boldsymbol{\theta}_{\boldsymbol{k}}}$ is the tomographic reconstructor for the direction $\boldsymbol{\theta}_{\boldsymbol{k}}, \boldsymbol{\varphi}_{\boldsymbol{k}}$ is the actual aperture-plane phase coming from the 
direction $\boldsymbol{\theta}_{\boldsymbol{k}}, \hat{\boldsymbol{\varphi}}_{\boldsymbol{k}}=\boldsymbol{E}_{\boldsymbol{\theta}_{\boldsymbol{k}}} \boldsymbol{s}$ is the aperture-plane phase estimated from slopes measured by SH-WFSs $\boldsymbol{s}$, and \langle\rangle indicates ensemble average over time. With Eq. (1), this minimization can be equivalent to the minimization of the variance of the total residual phase distortion in all $N_{l}$ layers,

$$
\boldsymbol{E}_{\boldsymbol{\theta}_{\boldsymbol{k}}}=\boldsymbol{P}_{\boldsymbol{\theta}_{\boldsymbol{k}}} \boldsymbol{E}_{\boldsymbol{s}}=\boldsymbol{P}_{\boldsymbol{\theta}_{\boldsymbol{k}}} \underset{\boldsymbol{E}_{\boldsymbol{s}}}{\operatorname{argmin}}\left\langle\|\boldsymbol{\phi}-\hat{\boldsymbol{\phi}}\|^{2}\right\rangle,
$$

where $\boldsymbol{E}_{\boldsymbol{s}}$ is the reconstructor providing the phase distortion in each layer.

The slope $\boldsymbol{s}_{\boldsymbol{\theta}_{j}}(t)$ measured by the $j$ th SH-WFS aiming the direction $\boldsymbol{\theta}_{\boldsymbol{j}}$ at time $t$ is defined as

$$
\boldsymbol{s}_{\boldsymbol{\theta}_{j}}(t)=\boldsymbol{\Gamma}_{\boldsymbol{\theta}_{j}} \boldsymbol{P}_{\boldsymbol{\theta}_{j}} \boldsymbol{\phi}(t)+\boldsymbol{\eta}_{\boldsymbol{\theta}_{j}}(t),
$$

where $\boldsymbol{\Gamma}_{\boldsymbol{\theta}_{j}}$ is a discrete phase-to-slope operator which converts phases into slopes, and $\boldsymbol{\eta}_{\boldsymbol{\theta}_{j}}(t)$ is a column vector of the noise in measurements of the $j$ th WFS. Concatenating Eq. (4) of $N_{g s}$ WFSs, we can obtain an equation connecting the phase distortion in $N_{l}$ atmospheric layers and the slope provided by $N_{g s}$ WFS as follows:

$$
\boldsymbol{s}(t)=\boldsymbol{\Gamma} \boldsymbol{P}_{g s} \boldsymbol{\phi}(t)+\boldsymbol{\eta}(t),
$$

where $\boldsymbol{s}(t)=\left[\boldsymbol{s}_{\boldsymbol{\theta}_{\mathbf{1}}}^{T} \cdots \boldsymbol{s}_{\boldsymbol{\theta}_{N_{g s}}}^{T}\right]^{T}, \boldsymbol{\Gamma}$ is a block diagonal matrix as $\boldsymbol{\Gamma}=\operatorname{diag}\left[\boldsymbol{\Gamma}_{\boldsymbol{\theta}_{1}}, \cdots, \boldsymbol{\Gamma}_{\boldsymbol{\theta}_{N_{g s}}}\right], \boldsymbol{P}_{g s}$ is a GS ray-tracing matrix which is a concatenation of $\boldsymbol{P}_{\boldsymbol{\theta}_{j}}$ for $1 \leq j \leq N_{g s}$, and $\boldsymbol{\eta}(t)$ is a column vector including measurement noises from all WFSs.

As shown in [16], the reconstructor can be obtained by minimizing the partial derivative of Eq. (3) with respect to $\boldsymbol{E}_{\boldsymbol{s}}$ :

$$
\begin{aligned}
\hat{\boldsymbol{\varphi}}_{\boldsymbol{\theta}_{\boldsymbol{k}}}(t) & =\boldsymbol{P}_{\boldsymbol{\theta}_{\boldsymbol{k}}} \boldsymbol{E}_{\boldsymbol{s}} \boldsymbol{s}(t) \\
& =\boldsymbol{P}_{\boldsymbol{\theta}_{\boldsymbol{k}}}\left(\boldsymbol{P}_{\boldsymbol{g}^{s}}^{T} \boldsymbol{\Gamma}^{T} \Sigma_{\boldsymbol{\eta}}^{-1} \boldsymbol{\Gamma} \boldsymbol{P}_{\boldsymbol{g} \boldsymbol{s}}+\boldsymbol{L}^{T} \boldsymbol{L}\right)^{-1} \boldsymbol{P}_{\boldsymbol{g} \boldsymbol{s}}^{T} \boldsymbol{\Gamma} \Sigma_{\boldsymbol{\eta}}{ }^{-1} \boldsymbol{s}(t),
\end{aligned}
$$

where $\Sigma_{\eta}$ is a noise covariance matrix which is a block diagonal matrix as $\Sigma_{\eta}=\left[\sigma_{\eta, \theta_{1}}^{2} \boldsymbol{I}, \cdots, \sigma_{\eta, \theta_{N_{0}}}^{2} \boldsymbol{I}\right]$ under the assumption that the measurement noise from the $j$ th WFS is a zero-mean Gaussian noise with a variance of $\sigma_{\eta, \theta_{i}}^{2}$ and that noises of all WFS subapertures are independent of each other. The term $\boldsymbol{L}^{T} \boldsymbol{L}$ is an approximation of the phase inverse covariance matrix, $\Sigma_{\phi}^{-1}$, presented by [16] and the scaling of $\boldsymbol{L}$ is given in [17]. While this approximated regularization matrix is sparse and incorporated into efficient sparse-matrix techniques, the approximated matrix does not regularize tip-tilt modes and other modes that are curvature-free [18]. The unregularized modes, mainly tip-tilt modes, may affect the tomographic error especially in the case with LGSs, where tip-tilt modes cannot be measured by the LGS.

In the classical tomographic reconstruction, the phase distortion on the turbulence layers is estimated from the measured slopes at one time-step. Therefore, $\boldsymbol{E}_{\boldsymbol{s}}$ is referred to as single time-step tomographic reconstruction in the remainder of this paper.

\section{B. Multi Time-Step Tomographic Reconstruction}

Our idea is to reduce the geometric tomographic error by increasing information of the atmospheric turbulence using the measurements at both the previous and current time-steps simultaneously. The frozen flow assumption allows us to consider the evolution of the atmospheric turbulence as the movements of the turbulence layers due to the winds. Under this assumption, it can be considered that the areas measured by WFSs at a previous time-step shift due to the winds with time, which is indicated by dashed circles in Fig. 1. Thanks to the spatial displacements due to the winds, the areas corresponding to the measurements at the previous time-step cover the uncovered and the unoverlapped areas at the current time-step.

If an atmospheric turbulence layer moves with a constant velocity $\boldsymbol{v}=\left(v_{x}, v_{y}\right)$, the movement of the phase distortion pattern with a position $x$ at $\Delta t$ previous time-step is written as

$$
\phi(x, t-\Delta t)=\phi(x+v \Delta t, t),
$$

where $\Delta t$ is the time difference between the previous and current time-steps. Considering this movement, we define the model connecting measurements at the previous time-step $(t-\Delta t)$ with the phase distortion due to the atmospheric turbulence at current time-step $t$,
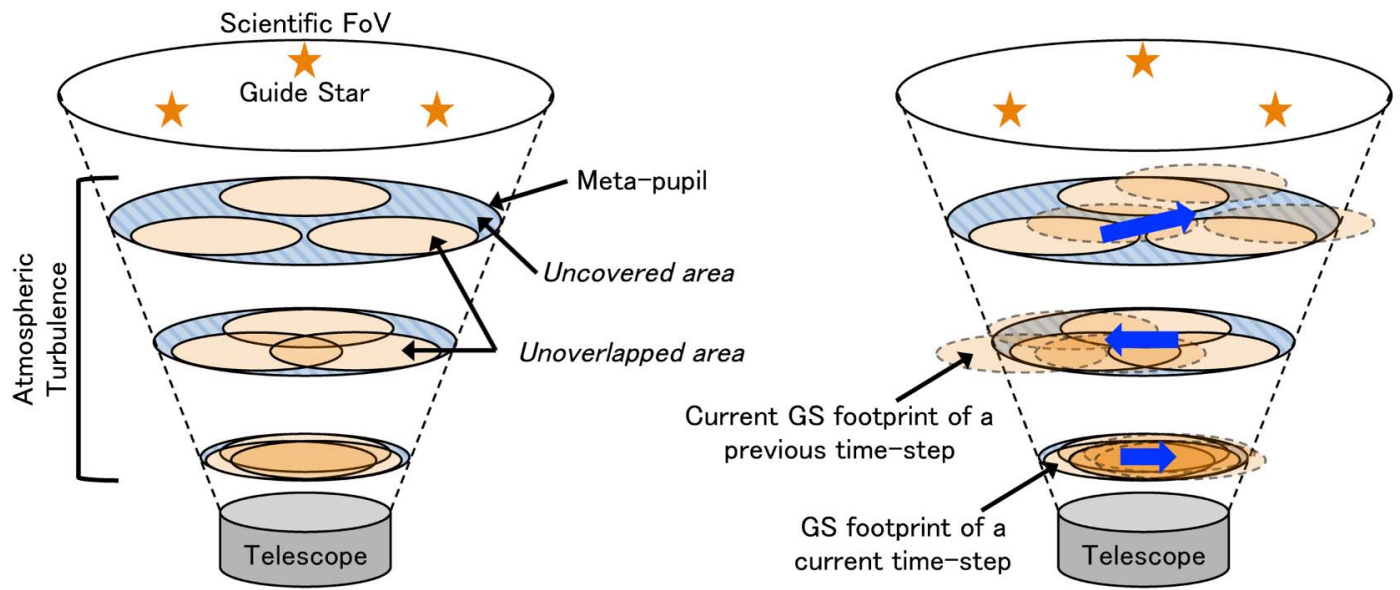

Fig. 1. Geometric relation between positions of GSs and regions in the atmosphere measured by WFSs. Left: the solid orange circles show the footprint of GS optical paths at the current time-step. There are areas covered by no or only one GS, referred to as uncovered area or unoverlapped area. These areas cause significant tomographic errors. Right: the blue arrows indicate wind direction at each altitude. The GS footprints measured at a previous time-step move due to the wind with time, which are dashed orange circles in the figure. Using the current and previous time-step measurements, which correspond to solid and dashed orange regions, we can reduce the uncovered and unoverlapped areas and improve the accuracy of a tomographic reconstruction. 


$$
\boldsymbol{s}(t-\Delta t)=\boldsymbol{\Gamma} \boldsymbol{P}_{\boldsymbol{g}^{s}}^{\Delta t} \boldsymbol{\phi}(t)+\boldsymbol{\eta}(t-\Delta t),
$$

where $\boldsymbol{P}_{g s}^{\Delta t}$ is a GS ray-tracing matrix considering the movement of the phase distortion pattern within the GS footprints during the duration time $\Delta t$. The reconstructor, which reconstructs the phase distortion from the measurements at both the current and previous time-steps, is given as the minimum variance solution of the concatenation of Eq. (5) with Eq. (8). The solution is given as in Eq. (6) as

$$
\begin{aligned}
\hat{\boldsymbol{\varphi}}_{\boldsymbol{k}}(t) & =\boldsymbol{P}_{\boldsymbol{\theta}_{\boldsymbol{k}}} \boldsymbol{E}_{\boldsymbol{m}} \overline{\boldsymbol{s}}(t) \\
& =\boldsymbol{P}_{\boldsymbol{\theta}_{\boldsymbol{k}}}\left(\overline{\boldsymbol{P}}_{\boldsymbol{g}}^{T} \overline{\boldsymbol{\Gamma}}^{T} \overline{\Sigma_{\eta}^{-1}} \bar{\Gamma} \overline{\boldsymbol{P}}_{\boldsymbol{g s}^{s}}+\boldsymbol{L}^{T} \boldsymbol{L}\right)^{-1} \overline{\boldsymbol{P}}_{\boldsymbol{g} \boldsymbol{s}}^{T} \bar{\Gamma} \bar{\Sigma}_{\boldsymbol{\eta}}^{-1} \overline{\boldsymbol{s}}(t),
\end{aligned}
$$

where

$$
\begin{gathered}
\overline{\boldsymbol{s}}=\left[\begin{array}{c}
\boldsymbol{s}(t) \\
\boldsymbol{s}(t-\Delta t)
\end{array}\right], \overline{\boldsymbol{P}}_{g^{s}}=\left[\begin{array}{c}
\boldsymbol{P}_{g_{s}} \\
\boldsymbol{P}_{g_{s}}^{\Delta t}
\end{array}\right], \\
\overline{\boldsymbol{\Gamma}}=\left[\begin{array}{cc}
\boldsymbol{\Gamma} & \mathbf{0} \\
\mathbf{0} & \boldsymbol{\Gamma}
\end{array}\right], \bar{\Sigma}_{\eta}=\left[\begin{array}{cc}
\Sigma_{\eta} & \mathbf{0} \\
\mathbf{0} & \Sigma_{\eta}
\end{array}\right] .
\end{gathered}
$$

Although the multi time-step reconstructor in Eq. (9) uses measurements only from two time-steps, it is easy to expand the reconstructor to use multi time-step measurements more than two time-steps.

The time difference between the current and previous timesteps, $\Delta t$, is an important parameter for the multi time-step reconstructor. Although we make the frozen flow assumption, in reality, the time scale in which the frozen flow assumption is valid is limited and the turbulence changes their structure with time, that is, the turbulence is boiling with time. Therefore, $\Delta t$ should be within the time scale in which the frozen flow assumption is valid. Otherwise, the multi time-step reconstructor does not work. Schöck and Spillar [19] found the intensity of the cumulative auto-correlation of SH-WFS measurements, including information from multiple turbulence layers, decreases to $90 \%$ of its initial value in $25 \mathrm{~ms}$ and to $50 \%$ in the range of 50 to $100 \mathrm{~ms}$. In this paper, we use this decay ratio as an indicator whether the frozen flow assumption is valid or not.

With respect to the computational complexity, the multi time-step reconstructor roughly doubles the size of the matrices. However, all matrices in the multi time-step reconstructor are sparse, and, therefore, a conjugate-gradient iterative scheme with preconditioners [20,21], warm-started wavefront reconstruction [22], and general purpose computing on GPU (GPGPU) can be used for accelerating the computation of the multi time-step reconstruction.

\section{Analytical Evaluation of Tomographic Error}

The tip-tilt removed tomographic error in the direction $\boldsymbol{\theta}_{\boldsymbol{k}}$ caused by a reconstructor $\boldsymbol{E}$ is defined as (see Appendix A)

$$
\begin{aligned}
\sigma_{\text {tomo }}^{2}\left(\boldsymbol{\theta}_{\boldsymbol{k}}\right)= & \left\langle\left\|\boldsymbol{\varphi}_{\boldsymbol{k}}-\hat{\boldsymbol{\varphi}}_{\boldsymbol{k}}\right\|^{2}\right\rangle / n_{\text {node }} \\
= & \boldsymbol{T r}\left[\boldsymbol{T} \boldsymbol{P}_{\boldsymbol{\theta}_{\boldsymbol{k}}}\left(\boldsymbol{I}-\boldsymbol{E} \boldsymbol{\Gamma} \boldsymbol{P}_{g_{s}}\right) \Sigma_{\boldsymbol{\phi}}\left(\boldsymbol{I}-\boldsymbol{E} \boldsymbol{\Gamma} \boldsymbol{P}_{g_{s}}\right)^{T} \boldsymbol{P}_{\boldsymbol{\theta}_{\boldsymbol{k}}}^{T} \boldsymbol{T}^{T}\right] / n_{\text {node }} \\
& +\boldsymbol{T r}\left[\boldsymbol{T} \boldsymbol{P}_{\boldsymbol{\theta}_{\boldsymbol{k}}} \boldsymbol{E} \Sigma_{\boldsymbol{\eta}} \boldsymbol{E}^{T} \boldsymbol{P}_{\boldsymbol{\theta}_{\boldsymbol{k}}}^{T} \boldsymbol{T}^{T}\right] / n_{\text {node }} \\
& =\sigma_{\text {geo }}^{2}+\sigma_{\text {noise }}^{2}
\end{aligned}
$$

where $n_{\text {node }}$ is the number of valid nodes on the aperture plane, $\boldsymbol{I}$ is an identity matrix, and $\boldsymbol{T}$ is a matrix removing a piston and tip-tilt modes. The first term $\sigma_{\text {geo }}$ represents an error depending on the geometry of GSs and the atmospheric turbulence. The influences from the uncovered and unoverlapped areas are included in the first term. The second term $\sigma_{\text {noise }}$ represents the propagation of the measurement noise through the tomographic reconstruction.

Equation (11) can be used for evaluating the tomographic error analytically. Here, we consider a very simple model of a MOAO system with a $30 \mathrm{~m}$ circular aperture, three NGSs on a ring with a radius from 20 arc sec up to 200 arc sec from the center of the scientific FoV, the Fried parameter $r_{0}$ is $0.156 \mathrm{~m}$, an outer scale $L_{0}$ is $30 \mathrm{~m}$, and three atmospheric turbulence layers at 0,5 , and $10 \mathrm{~km}$. The $C_{N}^{2}$ fractions are $[50 \%, 25 \%$, $25 \%]$, the wind speeds are $\left[5 \mathrm{~ms}^{-1}, 10 \mathrm{~ms}^{-1}, 20 \mathrm{~ms}^{-1}\right]$, and the wind directions are $\left[90^{\circ}, 0^{\circ}, 0^{\circ}\right]$. The size of the SH-WFS subaperture is $1 \mathrm{~m}$ on the aperture plane. We assume that all subapertures have the same measurement noise $\sigma_{\eta}$. Therefore, $\Sigma_{\eta}=\sigma_{\eta}^{2} I$. The spatial sampling of discrete grids on the turbulence layers and the aperture plane is $1 \mathrm{~m}$, which is same as the subaperture size.

The boiling of the turbulence is not taken into account in this analysis, but $\Delta t$ should be determined with considering the time scale in which the frozen flow holds. Since more than half of the turbulence still correlates at $50 \mathrm{~ms}$ as mentioned in Section 2.B, the frozen flow assumption may mostly or partially hold within this frame. We thus set $\Delta t=50 \mathrm{~ms}$ and will discuss the time scale in which the frozen flow is valid in more detail in Section 3.

Figure 2 shows $\sigma_{\text {tomo }}, \sigma_{\mathrm{geo}}$, and $\sigma_{\text {noise }}$ in the central direction computed by Eq. (11) with the single (the red solid lines in the figure) and multi (the blue dashed lines) time-step reconstructors with different asterism radii. For the single time-step reconstructor, the geometric error $\sigma_{\text {geo }}$ increases largely with the radius of the asterism due to the increase in the unoverlapped area and dominates the total tomographic error $\sigma_{\text {tomo. }}$. On the other hand, in the case of the multi time-step reconstructor, both the geometric error $\sigma_{\text {geo }}$ and the noise propagation $\sigma_{\text {noise }}$ increase with the asterism radius, but the errors are

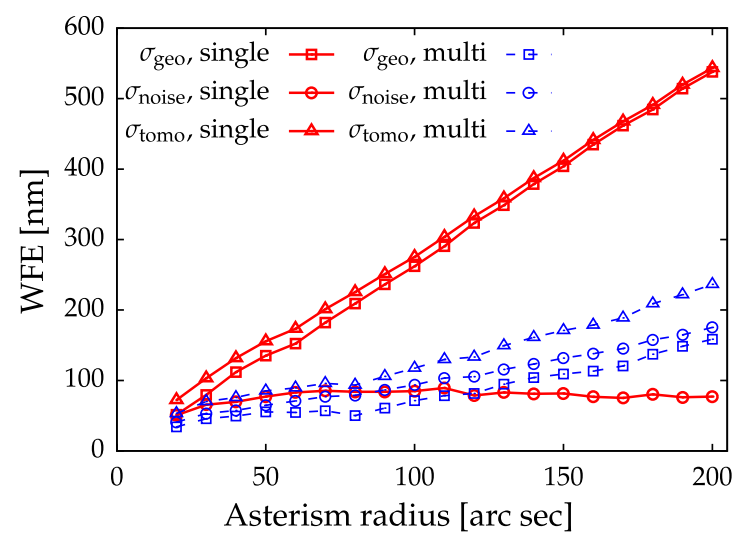

Fig. 2. Tomographic error $\sigma_{\text {tomo }}$ (triangles), geometric error $\sigma_{\text {geo }}$ (squares), and propagation of measurement noise $\sigma_{\text {noise }}$ (circles) computed by Eq. (11) with the single time-step reconstructor (the symbols with the red solid line) and the multi time-step reconstructor (the symbols with the blue dashed line). The fitting error is not included in the results. 


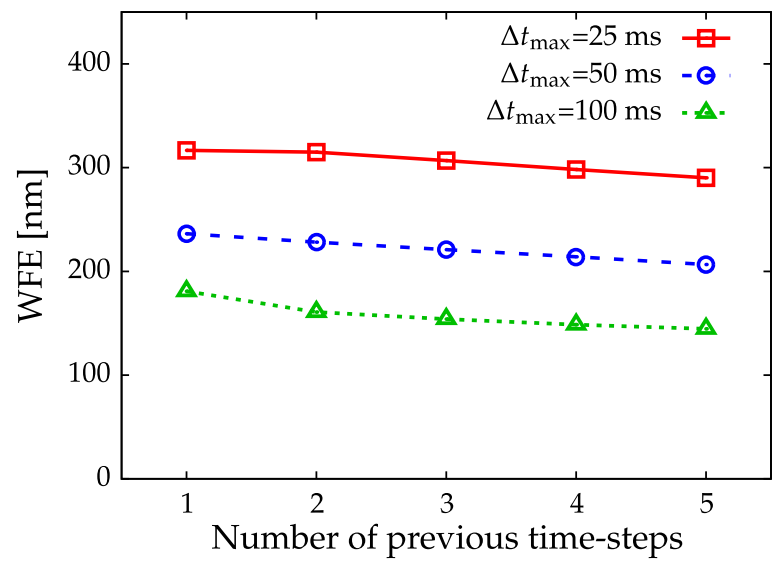

Fig. 3. Tomographic error $\sigma_{\text {tomo }}$ with the NGS asterism of a $200 \mathrm{arcsec}$ radius as a function of the number of previous time-steps, $N_{\Delta t}$. The variation of the line colors and types indicate the difference of the maximum $\Delta t$, which is denoted as $\Delta t_{\max }$. Each time difference is the integral multiple of $\Delta t_{\max } / N_{\Delta t}$. For example, if the number of the previous time-steps is 4 and $\Delta t_{\max }$ is $100 \mathrm{~ms}$, the time differences used in the multi time-step reconstruction are 25, 50, 75, and $100 \mathrm{~ms}$.

much smaller than $\sigma_{\text {geo }}$ of the single time-step reconstructor. Consequently, the multi time-step reconstructor reduces $\sigma_{\text {tomo }}$ especially at a larger asterism in comparison with the single time-step reconstructor. This result shows that the multi time-step reconstructor has a potential to reduce the tomographic error by using the measurement at previous time-steps and to enable the expansion of the size of scientific FoV of WFAO systems without reducing the correction performance. It is noted that this analytical tomographic error is based on the simple model and does not include the fitting error, the temporal lag-error, uncertainty of the atmospheric turbulence model, and uncertainties of the wind speeds and directions.

Figure 3 shows the dependency of the tomographic error $\sigma_{\text {tomo }}$ on the number of the previous time-steps, $N_{\Delta t}$. Different lines show the results with different maximum time differences, $\Delta t_{\max }$, used in the multi time-step reconstruction. The temporal sampling of $\Delta t$ is determined by $\Delta t_{\max } / N_{\Delta t}$. Namely, if $N_{\Delta t}$ is 4 and $\Delta t_{\max }$ is $100 \mathrm{~ms}$, the time differences used in the multi time-step reconstruction are $25,50,75$, and $100 \mathrm{~ms}$. The tomographic error decreases with the number of the previous time-steps for the same $\Delta t_{\max }$. Also, the tomographic error is reduced with longer $\Delta t_{\max }$. The gain with using longer $\Delta t_{\max }$ is larger than the gain with increasing the number of the time-steps, if the frozen flow assumption holds. In other words, the time scale in which the frozen flow assumption is valid has the most significant impact on the multi time-step reconstruction. We can reduce the tomographic error by increasing the number of the previous time-steps, as much as the computational complexity of the multi time-steps is acceptable.

\section{END-TO-END SIMULATION}

\section{A. Setting}

Parameters used in the E2E simulation are listed in Table 1. We assume a MOAO system with a scientific FoV with a
Table 1. Parameters Used in the Numerical Simulation

\begin{tabular}{lc}
\hline Parameters & Values \\
\hline Diameter of aperture & $30 \mathrm{~m}$ \\
scientific FoV & $10 \mathrm{arc} \mathrm{min}$ \\
Zenith angle & $0^{\circ}$ \\
Number of LGSs & 8 \\
Height of LGSs & $90 \mathrm{~km}$ \\
Number of turbulence layers & 7 \\
$r_{0}$ at $500 \mathrm{~nm}$ & $0.156 \mathrm{~m}$ \\
$L_{0}$ & $30 \mathrm{~m}$ \\
WFS & SH-WFS \\
Number of WFS subapertures & $60 \times 60$ \\
Number of DM elements & $60 \times 60$ \\
Frame rate & $800 \mathrm{~Hz}$ \\
Time lag & $2 \mathrm{frames}$ \\
Count per a subaperture & 700 electrons \\
Readout noise & 3 electrons \\
Evaluate wavelength & $1650 \mathrm{~nm}$ (H-band) \\
\hline
\end{tabular}

10 arc min diameter on a $30 \mathrm{~m}$ circular-aperture telescope with eight sodium LGSs at $90 \mathrm{~km}$. Two asterisms are used in the simulation, which are shown in Fig. 4. One is a narrow asterism, indicated as open squares in Fig. 4, and the other is a wide asterism, indicated as filled squares. Low-order modes of the phase distortion, which are tip, tilt, and focus, cannot be measured by an LGS due to uncertainty in LGS position on sky. Therefore, an NGS is required in a scientific FoV to measure these low-order modes when we use an LGS. In this simulation, however, we assume that we can measure the low-order modes of the phase distortion from the LGSs for simplicity. The cone effect and the spot elongation on SH-WFS are considered in the simulation. The sodium-layer profile is approximated to the lidar measurements used in [23] as

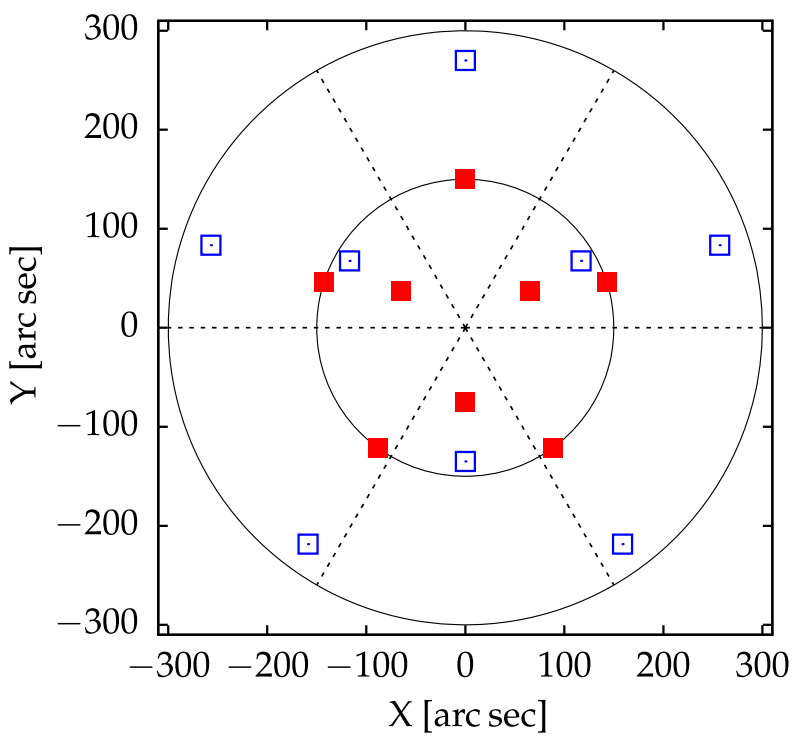

Fig. 4. LGS asterisms used in the numerical simulation. A narrow asterism is indicated with the red filled squares. The blue open squares show a wide asterism. The black solid lines show the $2.5 \mathrm{arc}$ min and 5 arc min radii from the center of a scientific FoV. The dashed lines are directions where the performance is evaluated. 
Table 2. Atmospheric Turbulence Profile Used in the Numerical Simulation

\begin{tabular}{lcccc}
\hline $\begin{array}{l}\text { Altitude } \\
{[\mathbf{k m}]}\end{array}$ & $\begin{array}{c}\boldsymbol{\int} \boldsymbol{C}_{\boldsymbol{N}}^{\mathbf{2}} \mathbf{d} \boldsymbol{b} \\
{\left[\mathbf{1 0}^{\mathbf{- 1 4}} \mathbf{~ m}^{\mathbf{1 / 3}}\right]}\end{array}$ & $\begin{array}{c}\text { Fraction } \\
\text { of } \boldsymbol{C}_{\boldsymbol{N}}^{\mathbf{2}}\end{array}$ & $\begin{array}{c}\text { Wind } \\
\text { Speed } \\
{\left[\mathbf{m s}^{-\mathbf{1}}\right]}\end{array}$ & $\begin{array}{c}\text { Wind } \\
\text { Direction } \\
{[\mathbf{d e g}]}\end{array}$ \\
\hline 16 & 2.734 & 0.0826 & 7.0 & 0 \\
8 & 2.264 & 0.0684 & 33.0 & 45 \\
4 & 2.879 & 0.0869 & 19.7 & 90 \\
2 & 1.233 & 0.0372 & 11.6 & 135 \\
1 & 1.074 & 0.0325 & 9.0 & 180 \\
0.5 & 3.190 & 0.0963 & 8.0 & 225 \\
0 & 19.737 & 0.5960 & 7.0 & 270 \\
Total $r_{0}[\mathrm{~m}]$ & 0.156 & & & \\
\hline
\end{tabular}

$$
\mathrm{Na}(h)=\left\{\begin{array}{cc}
\exp \left\{-\frac{\left(h-h_{\mathrm{LGS}}\right)^{2}}{2 \sigma_{\mathrm{Na}}^{2}}\right\} & \left(\text { for }\left|h-h_{\mathrm{LGS}}\right|<\sigma_{\mathrm{Na}}\right), \\
0 & \left(\text { for }\left|h-h_{\mathrm{LGS}}\right|>\sigma_{\mathrm{Na}}\right),
\end{array}\right.
$$

where $h_{\mathrm{LGS}}=88 \mathrm{~km}$ is the altitude of a sodium layer above the telescope, and $\sigma_{\mathrm{Na}}=5 \mathrm{~km}$ is the half width of the sodium layer. We use SH-WFSs with $60 \times 60$ subapertures and DMs for science targets with $60 \times 60$ elements.

We assume the top of Maunakea as the observation site in the simulation and use a seven-layer model used in [24] for an atmospheric turbulence profile. This model is created based on image-quality measurements from the Subaru Observatory [25], combined with differential image motion monitor (DIMM) and multiple aperture scintillation sensor (MASS) measurements by the Thirty Meter Telescope (TMT) Project at Maunakea [26]. The model includes the additional dome seeing in the Subaru Telescope. Thus, the ground layer has a strong turbulence power, which is $60 \%$ of the total turbulence power. The Fried parameter $r_{0}$ is in the 50 th percentile of the seeing measurements, $r_{0}=0.156 \mathrm{~m}$, and an outer scale is assumed to be $30 \mathrm{~m}$. The $C_{N}^{2}$ values at each altitude are summarized in Table 2. We assume a Gaussian model for the wind speeds on each layer based on [27]. The assumed wind speeds and directions are also listed in Table 2. The performance is evaluated at a wavelength of $1650 \mathrm{~nm}$ (H-band). The turbulence simulated in the simulation follows perfectly the frozen flow assumption.

\section{B. Simulation Results}

First, we simulated the optimal performance of the single and the multi time-step reconstructors for both the narrow and wide LGS asterisms with an assumption that the turbulence profile, wind speeds, and directions are known a priori. The results are illustrated in Fig. 5. SR values are measured from simulated point spread function (PSF) images by comparing the peak intensities of the PSF images to the peak intensity of a diffraction-limited PSF image created by the simulation. All PSF images from the simulation are normalized by the total intensity within a 1 arc sec box. The top and the bottom panels in Fig. 5 show the simulated maps of the Strehl ratio (SR) across the scientific FoV of 10 arc min diameter with the single (top) and multi time-step (bottom) reconstructors. We use $\Delta t$ of $50 \mathrm{~ms}$ for the multi time-step reconstructor as in Section 2. The bottom panels in Fig. 5 show SR profiles as a function of an angular distance from the center of the scientific FoV.
The profiles are computed by averaging over six directions shown as dashed lines in Fig. 4.

With the narrow asterism, the single time-step reconstructor, which is indicated as red filled squares in the left panels of Fig. 5, achieves an average SR of $\sim 0.5$ within the inner LGS radius at 75 arc sec from the center. The average SR value decreases slowly with an angular separation from 75 to 150 arc sec. In the region outside the 150 arc sec radius, the average SR values decrease steeply because there is no LGS. The multi time-step reconstructor with $\Delta t=50 \mathrm{~ms}$, represented as the blue open squares, can increase the SR value over the scientific FoV. The improvement factor of the average SR value is 1.25 at 150 arc sec, even though there is no uncovered area at the inner region below 150 arc sec. This suggests that the single time-step reconstructor is affected by the degeneracy due to the unoverlapped area even with the narrow asterism.

In the case of the wide asterism, illustrated in the right panels of Fig. 5, the SR value in the region outside the $200 \mathrm{arc} \mathrm{sec}$ radius is better than the value of the narrow asterism because the wider area is covered by the LGSs. In the contrast, the larger separation between LGSs causes a larger unoverlapped area at high altitudes. Hence, the SR value of the inside area becomes less than that of the narrow asterism, and there are valley areas between LGSs on the SR map (the left top panel of Fig. 5). From the bottom right panel of Fig. 5, the maximum average $\mathrm{SR}$ value is 0.24 with the single time-step reconstructor. This is affected strongly by unoverlapped area. Using the multi timestep reconstructor with $\Delta t=50 \mathrm{~ms}$, the average $\mathrm{SR}$ value is doubled over the scientific FoV for the wide asterism. At 300 arc sec from the center, the SR value from the multi time-step reconstructor with the wide asterism is three times larger than the result of the narrow asterism.

The performance of the multi time-step reconstructor depends on the spatial displacement between the areas measured by the current and previous time-steps at each altitude, which we refer as $d(h)$ in the paper. This displacement $d(h)$ corresponds to the movement of the atmospheric turbulence layer at an altitude $h$ during $\Delta t, d(h)=v(h) \Delta t$, with $v(h)$ as the wind speed at altitude $h$. The dependence of the multi time-step reconstructor on $\Delta t$ is presented in Fig. 6. The vertical axis in Fig. 6 is the SR improvement ratio, $k_{\mathrm{SR}}$, which represents the ratio of the SR value achieved by the multi time-step reconstructor to the SR value from the single time-step reconstructor and is averaged over the angular separation from the center of the scientific FoV. The multi timestep reconstructor achieves larger $k_{\mathrm{SR}}$ for the wide asterism than the narrow one. This suggests the wide asterism is affected more by the unoverlapped area than the narrow asterism. However, the trend of the dependence of $k_{\mathrm{SR}}$ on $\Delta t$ looks similar for both asterisms. This suggests that the dependence on $\Delta t$ does not depend the asterism of GSs. The multi time-step reconstructor improves the SR even with small $\Delta t$. The improvement ratio is 1.23 for $\Delta t=20 \mathrm{~ms}$ for the narrow asterism. The improvement ratio $k_{\mathrm{SR}}$ is maximized at $\Delta t \sim 100 \mathrm{~ms}$, where $k_{\mathrm{SR}} \sim$ 1.8 and $\sim 2.1$ for the narrow and the wide asterism, respectively. The displacements $d$ with $\Delta t=100 \mathrm{~ms}$ are $3.3 \mathrm{~m}$ and $0.7 \mathrm{~m}$ for the fastest and slowest layers in the model, respectively. While, as $\Delta t$ is larger than $100 \mathrm{~ms}, k_{\mathrm{SR}}$ has almost no or very 

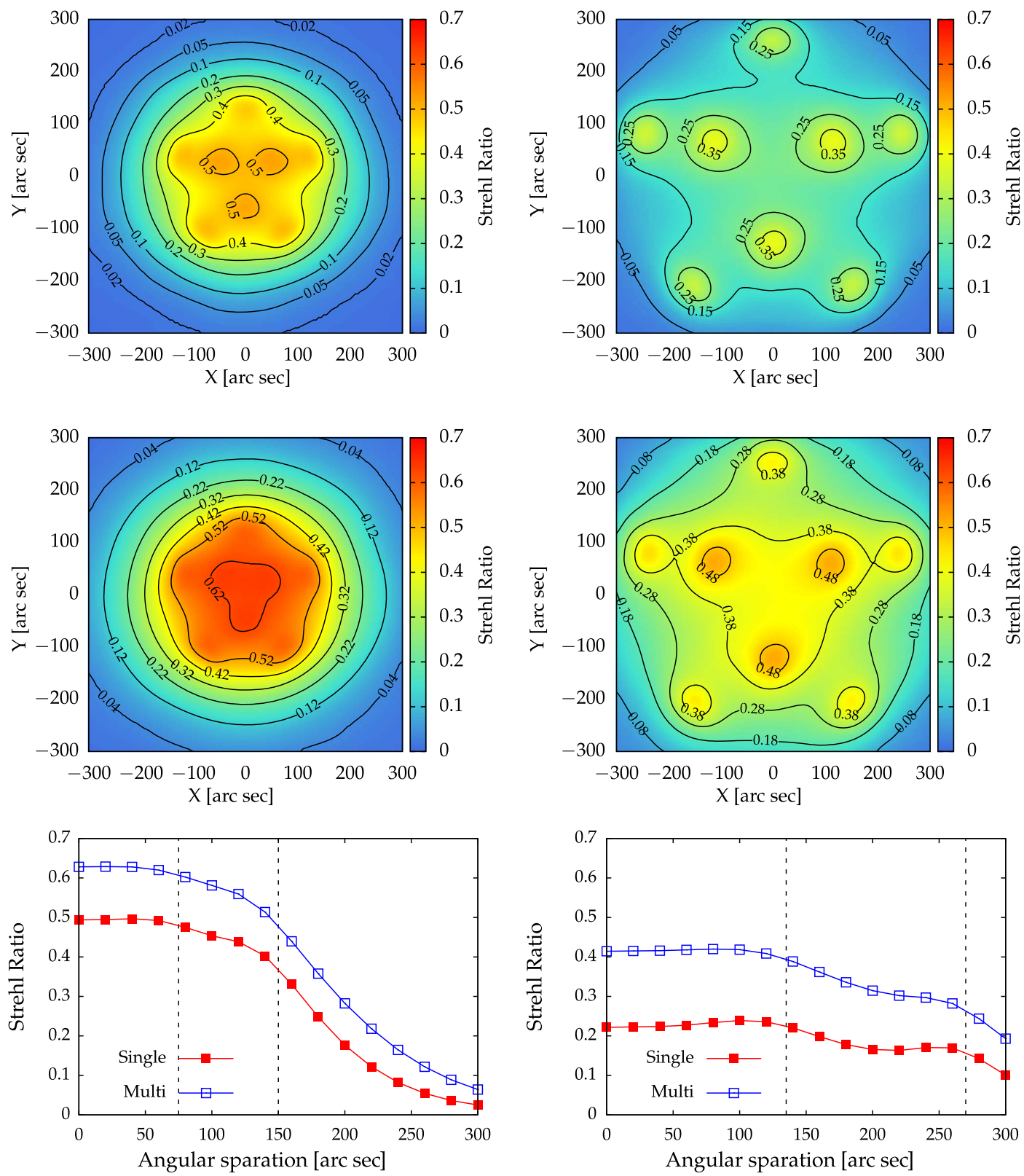

Fig. 5. Top and middle: simulated SR maps within the 10 arc min scientific FoV computed by using the single time-step reconstructor (top) or the multi time-step reconstructor with $\Delta t=50 \mathrm{~ms}$ (middle). The right panels are the results with the narrow LGS asterism, and the left panels are computed with the wide LGS asterism. The multi time-step reconstructor improves SR values over the scientific FoV for both of the asterisms. Bottom: SR profiles as a function of an angular separation from the center direction of the scientific FoV. The profiles are averaged over directions shown as the dashed lines in Fig. 4. The red filled squares show the averaged SR profile with the single time-step reconstructor, and the blue open squares are the result by the multi time-step reconstructor with $\Delta t=50 \mathrm{~ms}$. The dashed black lines show the radii of LGS positions. The SR values shown in this figure are evaluated including the tomographic error, the fitting error, and the temporal lag error. The turbulence used in the simulation follows perfectly the frozen flow.

week dependency on $\Delta t$. This suggests that only small displacements between the areas measured at the current and previous time-steps is enough to solve the degeneracy due to the unoverlapped area.

Figure 7 shows the dependency of the improvement ratio $k_{\mathrm{SR}}$ on the number of the previous time-steps, $N_{\Delta t}$. The improvement ratio increases with $N_{\Delta t}$, which is the same trend shown in the analytical evaluation (Fig. 3). Increasing the number of the previous time-steps provides an improvement in SR, if the computational complexity is acceptable.

The results shown above assume that the atmospheric turbulence follows perfectly the frozen flow assumption. As mentioned before, however, the time scale of the frozen flow assumption is valid only within a short time. Beyond this range the phase distortion pattern of the atmospheric turbulence varies. If the time scale of the frozen flow is smaller than $\Delta t$ 


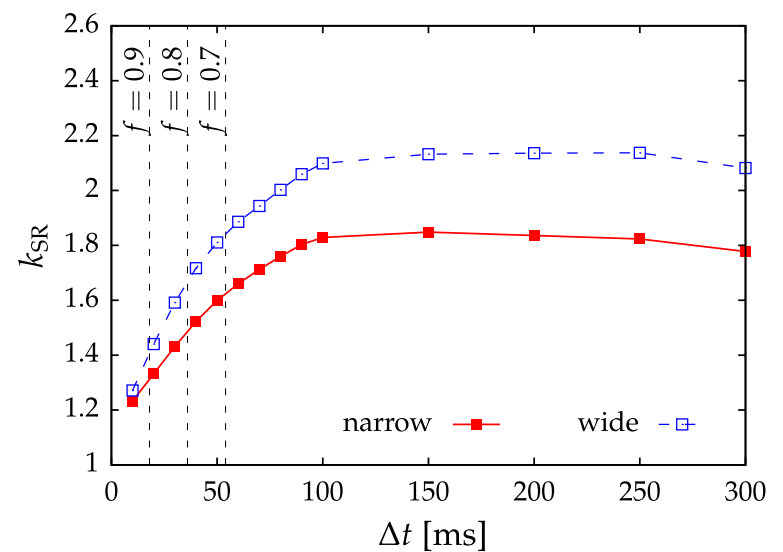

Fig. 6. Dependence of the multi time-step reconstructor on the $\Delta t$ is presented in Fig. 6. The vertical axis in Fig. 6 is the SR improvement ratio, $k_{\mathrm{SR}}$, which represents the ratio of the $\mathrm{SR}$ value achieved by the multi time-step reconstructor to the SR value from the single time-step reconstructor and is averaged over the angular separation from the center of the FoV. This SR improvement ratio includes the tomographic error, the fitting error, and the temporal lag error. The result with the narrow asterism is indicated as the red filled squares, and the blue open squares show the result with the wide asterism. The vertical dashed lines indicate the time duration that the decay ratio $f$ of the temporal correlation of SH-WFS measurements is $0.9,0.8$, and 0.7 when the wind speed is $33 \mathrm{~ms}^{-1}$, which is the maximum value in the wind model, estimated from Eq. (13).

for the multi time-step reconstructor, the measurement at the previous time-step can no longer be used for it is uncorrelated with the current measurement. Guesalaga et al. [14] investigated the time evolution of the atmospheric turbulence by using spatiotemporal cross-correlations of the measurements from multiple SH-WFSs installed in the Gemini South Multi-Conjugate Adaptive Optics System (GeMS). They found that the decay ratio of the correlation peak intensity for an individual layer, $f$, decreases with the time delay for the correlation, $\Delta t$, and depends on the distance traveled by the layer, $d$. In their paper, the decay ratio is approximated as a function of $\Delta t$ and $v$ as

$$
f=(-0.157 v-0.365) \Delta t+1 \text {. }
$$

By using this equation, we estimate $\Delta t$ to restrict the decay ratio $f$ to $0.9,0.8$, and 0.7 assuming the fastest wind speed in the model, $v=33 \mathrm{~ms}^{-1}$, which is plotted in Fig. 6 as vertical dashed lines. In order to keep the decay ratio greater than 0.7 for the fastest layer, $\Delta t$ should be smaller than $54 \mathrm{~ms}$. For the slower layer, required $\Delta t$ to make the decay ratio 0.7 gets larger. In this paper, we assume that the frozen flow assumption is valid when the decay ratio is greater than 0.7 and set $\Delta t$ to keep a decay ratio greater than 0.7. However, it is still unclear how the boiling of the turbulence affects the performance of the multi time-step reconstruction, which is not modeled in the simulation. In order to understand the limits of the frozen flow assumption and evaluate the effect of the temporal decoherence of the turbulence, on-sky experiments are necessary.

At the end of this section, we discuss the effect of uncertainties of wind speed and direction. The errors in wind speeds and

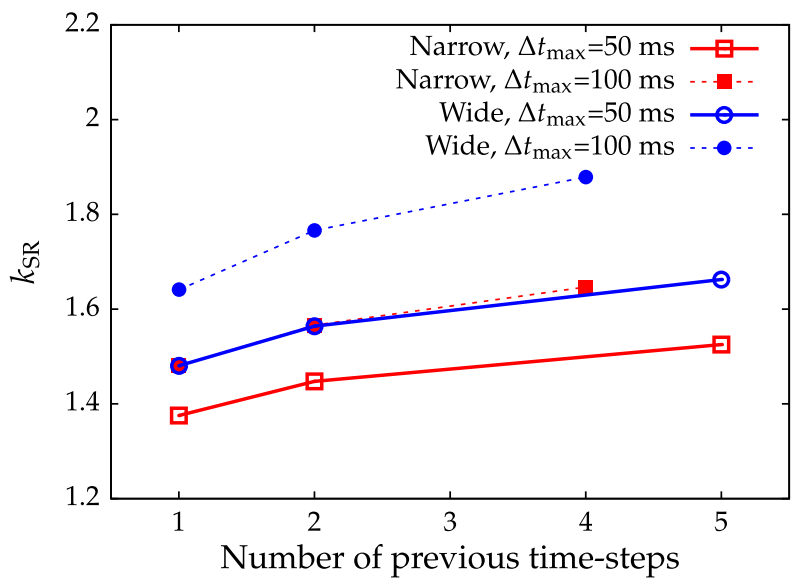

Fig. 7. Improvement ratio of $S R k_{\mathrm{SR}}$ as a function of the number of previous time-steps, $N_{\Delta t}$. The red circles show the result with the narrow asterism and the blue squares show the result with the wide asterism. The maximum time differences, $\Delta t_{\max }$, are set to $50 \mathrm{~ms}$ (the open symbols) and $100 \mathrm{~ms}$ (the filled symbols).

directions result in an error of the spatial displacement between the areas measured by the current and previous time-steps. Here, we represent the wind error as $\boldsymbol{e}=\left(e_{\|}, e_{\perp}\right)$. The first one, $e_{\|}$, is an error parallel to the wind direction and is referred to as wind speed error. The second, $e_{\perp}$, is an error perpendicular to the wind direction and is referred to as wind direction error. Figure 8 shows the improvement ratio $k_{\mathrm{SR}}$ achieved by the multi time-step reconstructor compared to the single time-step reconstructor with different errors of wind speed and direction, for both the narrow (red symbols) and wide (blue symbols) asterisms. We assume the same wind error for all atmospheric layers and $\Delta t=50 \mathrm{~ms}$ for the multi time-step reconstructor. The curve of $k_{\mathrm{SR}}$ with the wind direction error $e_{\perp}$ is symmetric

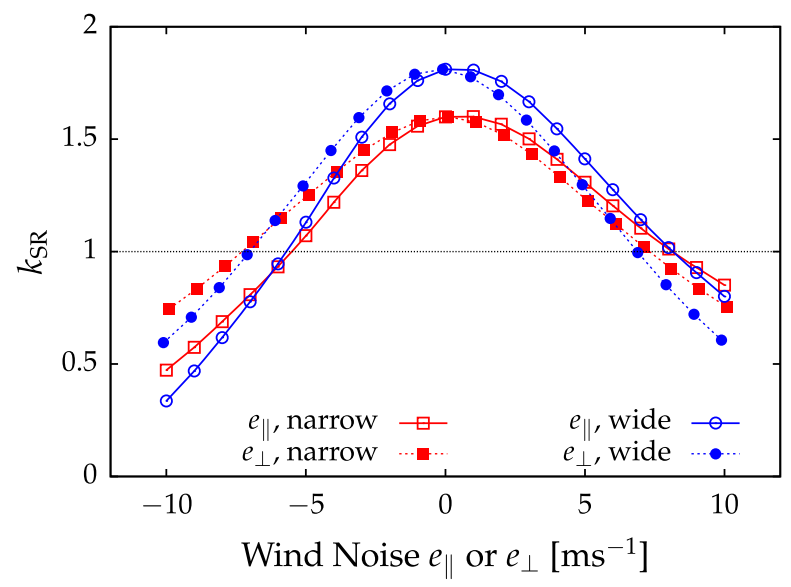

Fig. 8. SR improvement ratio, $k_{\mathrm{SR}}$, achieved by multi time-step tomographic reconstruction with different wind speed errors $e_{\|}$(the filled symbols) or wind direction errors $e_{\perp}$ (the open symbols). The results with the narrow asterism are represented as the red squares and the results with the wide asterism are the blue circles. The SR ratio of less than 1 means that the performance of the multi time-step reconstructor is poorer than the performance achieved by the single reconstructor due to the wind estimation error. 
with respect to the point $e_{\perp}=0$. The multi time-step reconstructor has the advantage compared to the singe time-step reconstructor, $k_{\mathrm{SR}}>0$, as $-7 \mathrm{~ms}^{-1} \leq e_{\perp} \leq 7 \mathrm{~ms}^{-1}$ for the condition we use for both asterisms. This corresponding spatial displacement error is less than $0.35 \mathrm{~m}$ and the error in angle of wind direction is less than $12 \mathrm{deg}$ for the fastest layer $\left(33 \mathrm{~ms}^{-1}\right)$ and $45 \mathrm{deg}$ for the slowest layer $\left(7 \mathrm{~ms}^{-1}\right)$ wind. For the wind speed error $e_{\|}$, the multi time-step reconstructor is affected more by negative $e_{\|}$, which is an error in the opposite direction to the wind, than positive $e_{\|}$. This is because if $e_{\|}$is negative, the covered areas are smaller than if $e_{\|}$is positive. The allowable range for $e_{\|}$is $-5.5 \mathrm{~ms}^{-1} \leq e_{\|} \leq 8 \mathrm{~ms}^{-1}$ for the wide asterism and $-5 \mathrm{~ms}^{-1} \leq e_{\perp} \leq 7 \mathrm{~ms}^{-1}$ for the narrow asterism. The dependence of the multi time-step reconstructor on the wind errors depends on the turbulence power, wind speed, direction, and $\Delta t$. If the turbulence powers are stronger, the influence of the uncertainty of wind speed and direction is larger. Using larger $\Delta t$ also makes the tomographic error due to the wind uncertainty larger because the spatial displacement error increases.

\section{ESTIMATION OF WIND SPEED AND DIRECTION}

As mentioned before, the altitude and power of the atmospheric turbulence layers (the turbulence profile) and the wind speeds and directions of multiple layers (the wind profile) are essential prior information for the multi time-step reconstruction. The turbulence profile can be estimated from the spatial cross-correlation of SH-WFS measurements by the SLOpe Detection and Ranging (SLODAR), which is a wellstudied method and already tested on sky [28-30]. We estimate the turbulence profile by the SLODAR proposed in [29].

The wind profile directions can be estimated by the spatiotemporal cross-correlation of the SH-WFS measurements [14]. The multiple peaks on the spatiotemporal cross-correlation map, which correspond to the multiple turbulence layers at different altitudes, move with the time delay for the spatiotemporal cross-correlation, depending on the wind speeds and directions. By tracking the correlation peaks, we can estimate the direction and speed of the wind of each turbulence layer. However, isolating and tracking the multiple peaks on the spatiotemporal correlation maps will be difficult when the peaks are not isolated, for example, the multiple peaks are overlapped along the tracks. In particular, in order to implement this wind estimation method to the real-time control, the method automatically needs to isolate and detect the multiple peaks.

In order to overcome this issue, we propose the tomographic wavefront reconstruction first to isolate the turbulence layer at different altitudes. Then, the spatiotemporal auto-correlation are applied to the isolated turbulence at each altitude to compute the wind speed and direction at its altitude. If the tomographic reconstruction works well, the spatiotemporal auto-correlation map of each turbulence layer has only one peak and we can track the peak more easily than the original spatiotemporal cross-correlation method. Furthermore, once the wind profile is estimated, we can use the multi time-step reconstruction to isolate each turbulence layer more clearly than the single time-step reconstruction and thus improve the accuracy of the wind estimation.

After the tomographic reconstruction provides the phase distortion of each turbulence layer, we extract aperture-size wavefronts at each altitude in the direction of the center of a GS asterism from the reconstructed phase distortions because the sizes of the turbulence layers are too large to compute spatiotemporal auto-correlation. In addition, it is better to use only areas reconstructed accurately for estimating wind speeds and directions. Although the GS directions have smaller integrated wavefront error (WFE) or better SR than other directions (see the SR maps in Fig. 5), it is possible that most areas at high altitude in the GS directions are covered only by one optical path, as shown in Fig. 1, and are affected by the degeneracy due to the unoverlapped area. This is because the reconstructed phase distortion at each altitude in the GS direction can cancel each other out and, as a result, the total WFE in this direction becomes smaller than other directions. Therefore, since the reconstructed phase distortion in the GS directions may not be isolated well, using the GS direction for the wind estimation is not optimal. As shown in Fig. 1, the direction of the center of the GS asterism is covered by multiple GS footprints, and the reconstructed phase distortion at each altitude is relatively accurate compared to the other directions.

We define a matrix $\boldsymbol{P}_{\boldsymbol{c}}$ as the cropping matrix, which extracts the wavefront at each altitude, not integrated wavefront, in the direction of the center of the GS asterism from the reconstructed phase distortion $\hat{\boldsymbol{\phi}}(t)=\left[\hat{\boldsymbol{\phi}}_{1}^{T}(t) \cdots \hat{\boldsymbol{\phi}}_{N_{l}}^{T}(t)\right]^{T}$, such that

$$
\hat{\boldsymbol{\phi}}_{\boldsymbol{c}}(t)=\left[\hat{\boldsymbol{\phi}}_{\boldsymbol{c}, 1}^{T}(t) \hat{\boldsymbol{\phi}}_{\boldsymbol{c}, 2}^{T}(t) \cdots \hat{\boldsymbol{\phi}}_{\boldsymbol{c}, \boldsymbol{N}_{\mathbf{1}}}^{T}(t)\right]^{T}=\boldsymbol{P}_{\boldsymbol{c}} \hat{\boldsymbol{\phi}}(t) .
$$

We perform the temporal auto-correlation of each turbulence layer in the slope space. The power spectral density (PSD) of the phase and slope have dependencies on the spatial frequency, $k$, as $k^{-11 / 3}$ and $k^{-5 / 3}$, respectively. Therefore, the temporal correlation of the slope tends to be more heightened at small time-delay and more long-lived at large separation than the phase due to the less steep PSD of the slope of phase. Thus, the peak of the slope correlation can be tracked easily compared with the peak of phase correlation. The conversion to the slope from the phase is performed by using the discrete phase-toslope operator, $\boldsymbol{\Gamma}_{\boldsymbol{c}}$, as $\hat{\boldsymbol{s}}_{\boldsymbol{c}, i}(t)=\boldsymbol{\Gamma}_{\boldsymbol{c}} \boldsymbol{T} \hat{\boldsymbol{\phi}}_{\boldsymbol{c}, \boldsymbol{i}}(t)$, where $\hat{\boldsymbol{s}}_{\boldsymbol{c}, \boldsymbol{i}}(t)$ is a reconstructed slope vector corresponding to a phase distortion of the $i$ th atmospheric turbulence layer in the direction of the center of the GS asterism at time $t$, and $\boldsymbol{T}$ is a matrix removing tip-tilt modes, which are affected by vibrations from the telescope and the instrument.

Temporal correlation of the reconstructed slope at the $i$ th turbulence layer can be computed as

$$
C_{i, x}(\delta u, \delta v, \delta t)=\frac{\left\langle\sum_{u, v} \hat{s}_{c, i, x}^{u, v}(t) \hat{\boldsymbol{s}}_{c, i, x}^{u+\delta u, v+\delta v}(t+\delta t)\right\rangle}{N(\delta u, \delta v)},
$$

where $u$ and $v$ are the subaperture indices along the $x$ and $y$ directions, respectively, $\hat{\boldsymbol{s}}_{\boldsymbol{c}, \boldsymbol{i}, \boldsymbol{v}}^{\boldsymbol{u} \boldsymbol{v}}(t)$ is an estimated $x$-direction slope vector in the subaperture $(u, v)$ at time $t, \delta u$ and $\delta v$ are relative distances between two subapertures, and $\delta t$ is the time delay for the temporal correlation. The summation, $\sum_{u, v}$, indicates the sum of all valid subaperture pairs with relative separation 
$(\delta u, \delta v), N(\delta u, \delta v)$ is the number of the pair with the separation $(\delta u, \delta v)$, and \langle\rangle represents average over time. Computing Eq. (15) for all possible pairs of $\Delta u$ and $\Delta v$, we can obtain a correlation map for the reconstructed $x$-direction slopes of the $i$ th turbulence layer. The correlation for the $y$-direction slopes is represented similarly to Eq. (15). In order to increase signal to noise, the correlations of the $x$ - and $y$-direction slopes are averaged, $C_{i}=\left(C_{i, x}+C_{i, y}\right)$.

The movement of the correlation peak on the correlation map $C_{i}$ with $\delta t$ corresponds to the wind speed and direction of the $i$ th turbulence layer. We use the centroid algorithm to detect the peak. With a SH-WFS subaperture size $d_{\text {sub }}$ and the peak position as $(\Delta u, \Delta v)$, the wind speed is computed by $v_{x}=$ $d_{\text {sub }} \Delta u / \delta t$ and $v_{y}=d_{\text {sub }} \Delta v / \delta t$. By computing $v_{x}$ and $v_{y}$ and averaging each one over different $\delta t$, the wind speed and direction at each altitude can be estimated.

\section{LABORATORY TEST WITH RAVEN}

\section{A. RAVEN}

RAVEN is a MOAO technical and science demonstrator installed and tested on the Subaru Telescope, which has a $8 \mathrm{~m}$ primary mirror, at Maunakea [15]. RAVEN can apply $\mathrm{MOAO}$ correction simultaneously for two science targets by tomographic reconstruction with three NGSs and one LGS attached to the Subaru Telescope [1]. The system of RAVEN is summarized in [15].

RAVEN has four open-loop SH-WFSs (OL-WFS) with $10 \times 10$ subapertures in the system, which are for three NGSs and one LGS. Each science optical path contains an ALPAO DM with $11 \times 11$ actuators. RAVEN has a calibration unit $(\mathrm{CU})$ on the optical bench, which simulates multiple GSs and science targets, three turbulence layers, and a telescope to calibrate and test the AO system [31]. Two high layers at altitudes of 10 and $5 \mathrm{~km}$ are simulated by phase screens, which can be rotated to simulate a movement of the turbulence layers due to the wind. The ground layer at $0 \mathrm{~km}$ is generated by a calibration DM with $17 \times 17$ actuators. The turbulence parameters simulated by the $\mathrm{CU}$ are summarized in the left panel of Fig. 10 and the top table of Table 3. It should be noted that the phase distortion patterns generated by the $\mathrm{CU}$ are periodic, and the period is $40 \mathrm{~s}$ for the $5 \mathrm{~km}$ layers and $19 \mathrm{~s}$ for the $10 \mathrm{~km}$ layer. RAVEN also has a near-infrared camera on the optical bench for laboratory experiments.

We test the wind estimation method and the multi timestep reconstruction with the $\mathrm{CU}$ and the infrared camera installed in RAVEN. An asterism that we used is shown in Fig. 9. The NGSs are at approximately a 60 arc sec radius. The brightness of the NGS is set to $R \sim 8 \mathrm{mag}$, which is very bright, and the WFS measurement error is minimal. The frame rate of the control is $250 \mathrm{~Hz}$.

\section{B. SLODAR and Wind Estimation}

Figure 10 and Table 3 show comparisons between the expected and estimated turbulence profile. The profile estimated by the SLODAR has three major peaks at the almost same altitudes as that of the expected profile. The estimated power of the ground layer is weaker than the expected value. As a result, the estimated total $r_{0}$ of $0.182 \mathrm{~m}$ is larger than the expected $r_{0}$ of
Table 3. Atmospheric Turbulence Profile and Wind Profile Generated by the RAVEN CU (Top) and Estimated by the SLODAR and the Wind Estimation Method (Bottom)

\begin{tabular}{|c|c|c|c|c|}
\hline \multicolumn{5}{|c|}{ Expected Profile } \\
\hline \multirow{2}{*}{$\begin{array}{l}\text { Altitude } \\
{[\mathrm{km}]}\end{array}$} & \multirow{2}{*}{$\underset{\left[10^{-14} \mathrm{~m}^{1 / 3}\right]}{C_{N}^{2} \mathrm{~d} h}$} & \multirow{2}{*}{$\begin{array}{c}\text { Fraction } \\
\text { of } C_{N}^{2}\end{array}$} & \multicolumn{2}{|c|}{ Wind Speed $\left[\mathrm{ms}^{-1}\right]$} \\
\hline & & & $x$ & $y$ \\
\hline 10.5 & 5.961 & 0.180 & 17.0 & 0.0 \\
\hline 5.5 & 7.418 & 0.224 & 6.0 & 0.0 \\
\hline 0 & 19.737 & 0.596 & 0.0 & 5.68 \\
\hline Total $r_{0}[\mathrm{~m}]$ & 0.156 & & & \\
\hline \multicolumn{5}{|c|}{ Estimated Profile } \\
\hline \multirow{2}{*}{$\begin{array}{l}\text { Altitude } \\
{[\mathrm{km}]}\end{array}$} & \multirow{2}{*}{$\begin{array}{c}\int C_{N}^{2} \mathrm{~d} h \\
{\left[10^{-14} \mathrm{~m}^{1 / 3}\right]}\end{array}$} & \multirow{2}{*}{$\begin{array}{l}\text { Fraction } \\
\text { of } C_{N}^{2} \\
\end{array}$} & \multicolumn{2}{|c|}{ Wind Speed $\left[\mathrm{ms}^{-1}\right]$} \\
\hline & & & $x$ & $y$ \\
\hline 10 & 4.329 & 0.169 & 15.4 & -0.6 \\
\hline 8 & 0.896 & 0.035 & 8.6 & -0.2 \\
\hline 6 & 8.222 & 0.321 & 6.3 & 0.1 \\
\hline 0 & 12.140 & 0.474 & 0.1 & 5.0 \\
\hline Total $r_{0}[\mathrm{~m}]$ & 0.182 & & & \\
\hline
\end{tabular}

$0.156 \mathrm{~m}$. Larger estimated $r_{0}$ than the designed value is already reported in the previous estimation in the laboratory on RAVEN [15]. Since the actuator size of the calibration DM in the $\mathrm{CU}$ is roughly $0.47 \mathrm{~m}$, the $\mathrm{DM}$ cannot reproduce the phase distortion with spatial scales smaller than $0.47 \mathrm{~m}$. We conclude that the actuator size causes the discrepancy and that the estimated value reflects the real turbulence profile on the DM better than the designed value.

By using the estimated profile, we evaluate the wind speed and direction at each layer. First, we compute the tomographic reconstruction to reconstruct the phase distortion pattern at each altitude. Then, the temporal correlation map of the estimated slopes are calculated with different delay times, $\delta t$. The temporal correlation maps are illustrated in Fig. 11. The delay time for the lower layers at 0 and $6 \mathrm{~km}$ are set larger than the higher layers (Fig. 11). Generally, the wind speed at low altitude is considered slower than the wind speed at high altitude. In order to get clear movement of the temporal correlation peak, longer delay time is preferred for the low altitude layers. For all

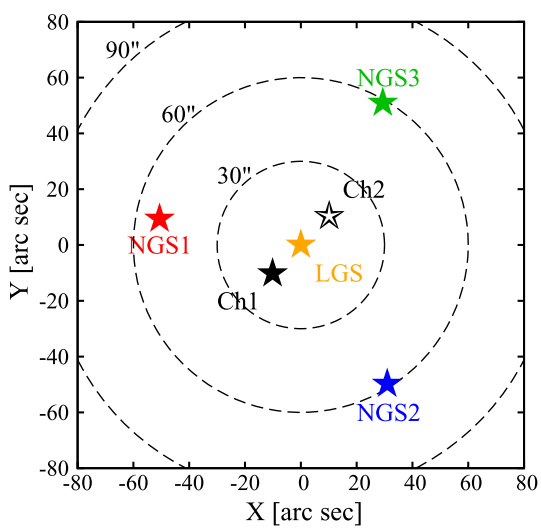

Fig. 9. Asterism of the GSs and the science targets for the laboratory test with the RAVEN. 

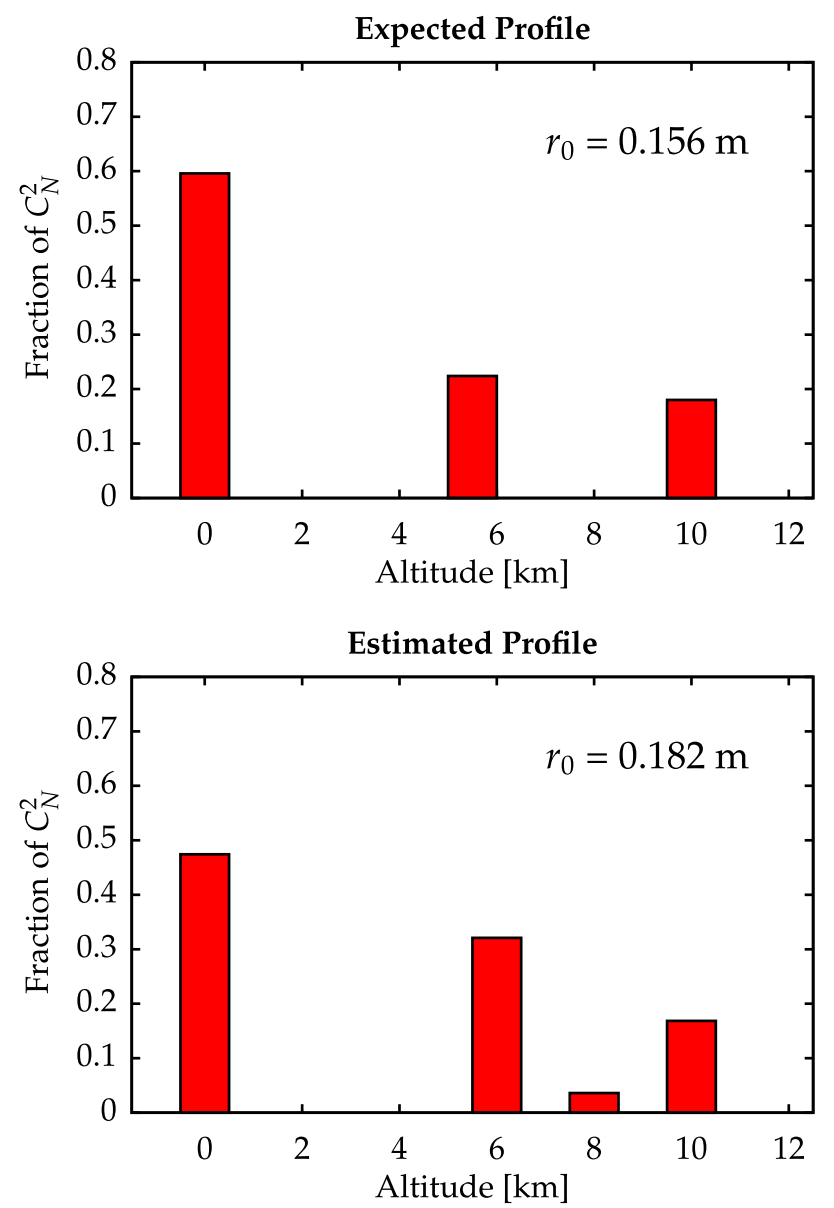

Fig. 10. Left: the expected turbulence profile generated by the RAVEN CU. Right: the turbulence profile estimated by the SLODAR in the laboratory on RAVEN.

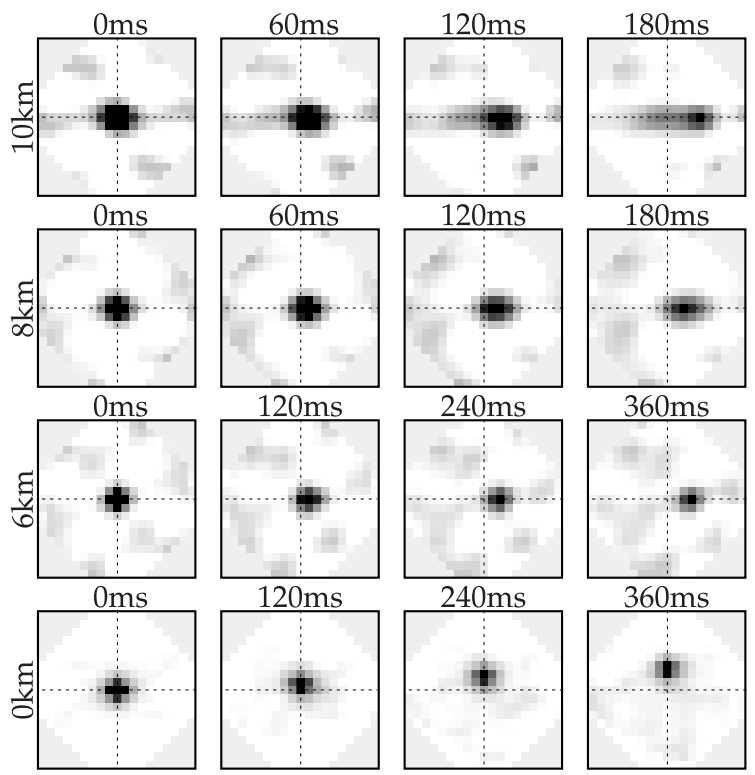

Fig. 11. Temporal correlation maps of the slope at each altitude estimated by tomographic reconstruction. The values at the top of each image indicate the time delay used to drive the temporal correlation. layers detected by the SLODAR, the peaks on the temporal correlation maps are detected. The estimated wind speeds and directions, which are summarized in the bottom table of Table 3, are consistent with the expected value. The error of the wind speed is less than $2 \mathrm{~ms}^{-1}$ for all layers, which is an acceptable wind error computed by the numerical simulation in Section 3. With this wind estimation error, the multi time-step reconstructor will work with almost maximum performance on the future MOAO system (see Fig. 8).

We also compute the wind speeds and directions by the method proposed in [14] as a comparison. The temporal cross-correlation maps between the slopes from SH-WFS2 and SH-WFS3 are shown in Fig. 12. There are three peaks, moving with $\delta t$, on the temporal cross-correlation maps. The altitudes corresponding to these peaks are 0,6 , and $11 \mathrm{~km}$, respectively. The wind speeds and directions are estimated by tracking these peaks. The estimated wind speeds in the $x$ and $y$ directions are $[-0.1,4.7] \mathrm{ms}^{-1}$ at $0 \mathrm{~km},[6.6,0.0] \mathrm{ms}^{-1}$ at $6 \mathrm{~km}$, and $[17.4,3.0] \mathrm{ms}^{-1}$ at $11 \mathrm{~km}$. These values are consistent with the expected values and the values estimated by our wind estimation. In this laboratory test, there is no overlap of the peaks along tracks; thus, tracking the peaks is not difficult. However, if there are more peaks or/and there are overlaps of the peaks, tracking the peaks becomes more difficult. On the other hand, since the temporal correlation map of the reconstructed slopes (Fig. 11) provides only one peak in their maps, our method makes tracking the peaks easier. This is important to implement the wind estimation automatically.

\section{Multi Time-Step Reconstruction}

We present the result of the laboratory experiment of the multi time-step reconstruction in Fig. 13 and the top table of Table 4. Figure 13 shows the PSF images of two science channels taken by the RAVEN infrared camera with a MOAO correction. The ensquared energy (EE) values included in a 140 mas box and $k_{\mathrm{SR}}$ computed from the PSF images are summarized in Table 4 . The maximum wind speed simulated by the $\mathrm{CU}$, which is $17.0 \mathrm{~ms}^{-1}$, is almost half of the maximum wind speed in the model that we used for the numerical simulation in Section 3, and the time duration constraining the decay ratio $f$ of the temporal correlation of SH-WFS measurements to greater than 0.7 for a wind speed of $17.0 \mathrm{~ms}^{-1}$ is $\sim 100 \mathrm{~ms}$. Therefore, we use $100 \mathrm{~ms}$ for $\Delta t$ of the multi time-step reconstructor. The pixel scale of the PSF image is 17.5 mas, and the size of an image is $0.5 \mathrm{arcsec} \times 0.5 \mathrm{arc} \mathrm{sec}$. The performance of the MOAO is evaluated by $S R, k_{S R}$, and an EE at wavelength of $1650 \mathrm{~nm}$ (H-band), which is a ratio of intensity within a defined square to the total intensity. We use the simulated diffraction-limited PSF image to measure SR values of the PSF images taken in the laboratory test. The laboratory test PSF images are normalized by the total intensity within a 1 arc sec box, which is the same as the simulated PSF images. The size of a square for the EE is set to 140 mas, which is the size of the slit of the IRCS.

Visually, the PSFs become sharper and have a higher peak intensity with the multi time-step reconstruction compared to the single time-step reconstruction for both of the asterisms, as 


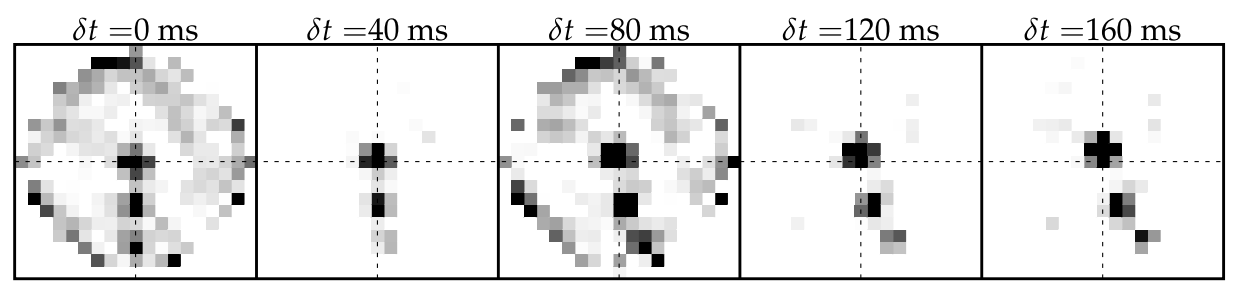

Fig. 12. Temporal cross-correlation map between the slopes from SH-WFS2 and SH-WFS3. This map is used for the wind estimation proposed in [14]. There are three peaks, moving with $\delta t$, on the temporal cross-correlation map. The altitudes corresponding to these peaks are 0 , 6 , and $11 \mathrm{~km}$, respectively. The wind speeds and directions are estimated by tracking these peaks. The estimated wind speeds in the $x$ and $y$ directions are $[-0.1,4.7] \mathrm{ms}^{-1}$ at $0 \mathrm{~km},[6.6,0.0] \mathrm{ms}^{-1}$ at $6 \mathrm{~km}$, and $[17.4,3.0] \mathrm{ms}^{-1}$ at $11 \mathrm{~km}$. These values are consistent with the expected values and the values estimated by the wind estimation proposed in this paper.
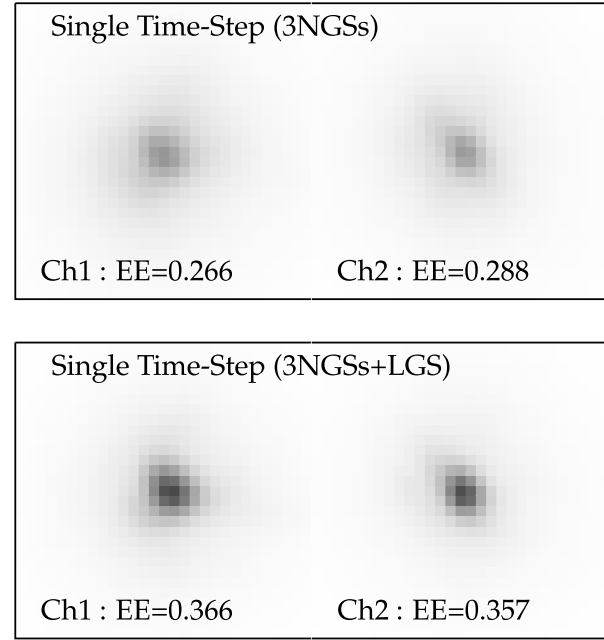
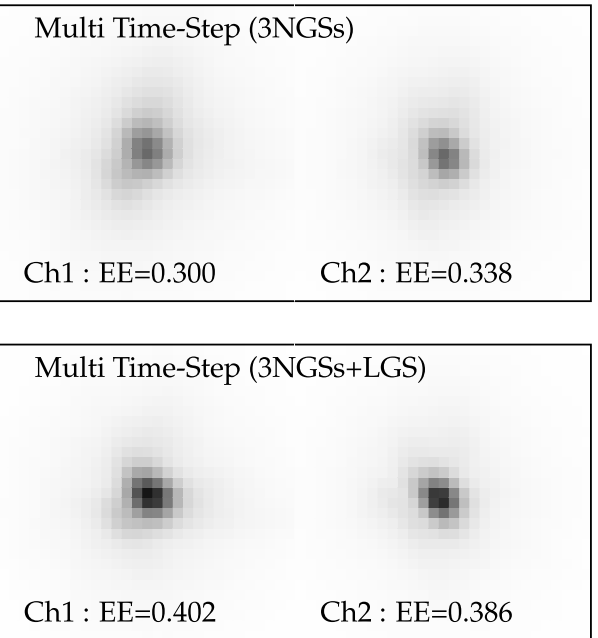

Fig. 13. PSF images of each science channel taken in the laboratory test with the single and multi time-step reconstructors. The color scale is linear and aligned for each channel. The wavelength is $1650 \mathrm{~nm}$ (H-band).

shown in Fig. 13. The EEs achieved by the single time-step reconstructor are $\sim 0.28$ for the asterism without LGS and $\sim 0.35$ with LGS. The improvement ratios of the EE achieved by the multi time-step reconstructor are 1.05-1.18. The absolute increases of the EE are $0.03-0.05$. The $S R$ is $\sim 0.08$ with the single time-step reconstructor without the LGS, and this is consistent with the result of 0.10 measured in the laboratory experiment with the RAVEN CU previously [15]. On the other hand, the SR is $\sim 0.135$ with the LGS, which is better than the one without LGS but lower than the previous value of 0.23 [15]. The improvement ratio, $k_{\mathrm{SR}}$, is $\sim 1.4$ without LGS and $\sim 1.23$ with LGS. This result means the multi time-step reconstruction works well in the laboratory experiment.

\section{DISCUSSION}

We compare the results of the laboratory experiment using the multi time-step reconstructor to an E2E numerical simulation. We assume that the parameters of the RAVEN system and the turbulence profile generated by the RAVEN CU, listed in the top table of Table 3. The GS and the target coordinates are also set to the same as the coordinates used in the laboratory test, as illustrated in Fig. 9. The EE values and the improvement ratio, $k_{\mathrm{SR}}$, predicted by the simulation are summarized in the lower part of Table 4 . There is a good agreement between the $k_{\mathrm{SR}}$ measured in the laboratory test and derived by the simulation. Therefore, the performance improvement of the multi timestep reconstructor is as predicted for both asterisms with and without LGS.

However, the EE and the SR measured in the laboratory test are much smaller than the values predicted by the simulation for both the single and multi time-step reconstructors. This difference may be due to implementation errors not considered in the simulation, which are calibration error, DM control error, and an optical aberration. The improvement ratio, $k_{\mathrm{SR}}$, is not affected by the implementation errors. Anderson et al. (2012) [24] simulates the performance of the RAVEN and estimates the error budget in the RAVEN system. They estimate a WFE caused by the implementation errors of RAVEN, which includes calibration error, DM flattening, DM stability, DM repeatability, and a high-order optical error, which is $107 \mathrm{~nm}$. The WFE due to the tomographic error is estimated from the SR derived from the simulation by using an approximation, $\mathrm{SR}=\exp \left[-\sigma^{2}\right]$, where $\sigma=2 \pi \mathrm{WFE} / \lambda$ and $\lambda=1650 \mathrm{~nm}$. It is noted that this expression is valid for $\sigma^{2}<1 \mathrm{rad}^{2}$, this is $\mathrm{SR}<0.37$, and our result can be biased when SR is lower than 0.37 . The total WFE is a quadrature 
Table 4. SR, $k_{S R}$, and EE Value of Each Channel Defined by a 140 mas Box Measured in the Laboratory Test (Top) Compared to Those Predicted from the Numerical Simulation (Bottom) ${ }^{a}$

\begin{tabular}{|c|c|c|c|c|c|}
\hline \multicolumn{2}{|c|}{ Laboratory Test } & \multicolumn{2}{|c|}{$\mathrm{EE}(140 \mathrm{mas})$} & \multicolumn{2}{|c|}{ SR } \\
\hline Single & $3 \mathrm{NGSs}$ & 0.268 & 0.290 & 0.072 & 0.087 \\
\hline Improvement ratio & & 1.16 & 1.18 & 1.32 & 1.51 \\
\hline Single & $3 \mathrm{NGSs}$ & 0.349 & 0.365 & 0.125 & 0.150 \\
\hline Multi & $+\mathrm{LGS}$ & 0.367 & 0.385 & 0.153 & 0.187 \\
\hline \multicolumn{2}{|c|}{ Simulation } & \multicolumn{2}{|c|}{ EE (140 mas) } & \multicolumn{2}{|c|}{ SR } \\
\hline reconstructor & GSs & Ch1 & $\mathrm{Ch} 2$ & Ch1 & Ch2 \\
\hline Single & 3NGSs & $0.334(0.294)$ & $0.346(0.303)$ & $0.251(0.211)$ & $0.267(0.224)$ \\
\hline Multi & & $0.406(0.348)$ & $0.424(0.362)$ & $0.361(0.302)$ & $0.384(0.321)$ \\
\hline Improvement ratio & & $1.21(1.18)$ & $1.23(1.19)$ & $1.44(1.43)$ & $1.44(1.43)$ \\
\hline
\end{tabular}

${ }^{a}$ There are two GS configurations used in the test, one is only three NGSs and the other is three NGSs+LGS. The values in parentheses in the lower part is the value accounting for the implementation errors.

sum of WFE due to the implementation errors and the tomographic error. We assume that the loss of the $\mathrm{EE}$ due to the implementation errors is the same as the loss of the SR. The performances predicted by simulation, accounting for the implementation errors, are listed in the bottom table of Table 4 as the values in parentheses. While the simulated EE including the implementation errors is slightly higher than the EE values measured in the laboratory test, the simulated SR including the implementation error is still much higher than the measured SR. This suggests that there is an additional error, which makes a PSF peak blurred within a 140 mas box and affects mostly SR values.

\section{CONCLUSIONS}

We propose the multi time-step reconstruction and a method estimating wind speeds and directions of multiple turbulence layers for tomographic WFAO systems. The multi time-step reconstruction is based on the frozen flow assumption and reduces the tomographic error caused by uncovered areas and degeneracy due to unoverlapped areas by increasing information of the atmospheric turbulence for the tomography by using the measurements at both of the previous and current time-steps simultaneously. The wind estimation method estimates wind speed and direction at each altitude by using temporal correlation of the phase distortion pattern reconstructed by the tomographic reconstruction.

The numerical simulation, assuming a MOAO system on a $30 \mathrm{~m}$ aperture telescope with eight LGSs at the top of Maunakea, shows that the multi time-step reconstructor with the time difference between the current and the previous timestep $\Delta t=50 \mathrm{~ms}$ increases SR over a scientific FoV of 10 arc min diameter by a factor of 1.5-1.8, depending on the guide star asterism, compared to the classical tomographic reconstructor if wind speeds and directions are given. Considering the time scale within which the frozen flow assumption is applicable, we set the time difference $\Delta t$ to keep the decay ratio of the peak intensity of temporal correlation to be more than 0.7. This is $\Delta t=54 \mathrm{~ms}$ for the fastest layer with $33 \mathrm{~ms}^{-1}$ wind in the atmospheric model that we use for the simulation. The acceptable ranges for the wind estimation error are $-7 \mathrm{~ms}^{-1} \leq e_{\perp} \leq 7 \mathrm{~ms}^{-1}$ for the wind direction error, $e_{\perp}$, and $-5.5 \mathrm{~ms}^{-1} \leq e_{\|} \leq 8 \mathrm{~ms}^{-1}$ for the wind speed error.

We test the multi time-step reconstruction and the wind estimation method in the laboratory test with the RAVEN $\mathrm{CU}$. The wind speeds and directions at multiple layers are estimated successfully in the laboratory test with the estimation error less than $2 \mathrm{~ms}^{-1}$. The improvement ratio of $\mathrm{EE}$ thanks to the multi time-step reconstruction is $1.05-1.18$ and the improvement ratio of $S R$ is 1.17-1.44, depending on the GS asterism. This improvement is consistent with the prediction from a numerical simulation if we include the WFE due to the implementation of the $\mathrm{AO}$ system.

The multi time-step reconstructor will bring a potentially significant gain for MOAO instruments designed for the future ELTs, MOSAIC for European ELT [6] and TMT-AGE for TMT [9,32]. The MOSAIC will use six LGSs at 7.5 arc min from the axis and up to five additional NGSs inside of that field, depending upon availability. The TMT-AGE will have eight LGSs within a scientific FoV of 10 arc min diameter. With these large LGS separations, the uncovered and unoverlapped area might significantly affect the final performance, even though the telescope aperture is large.

The main assumption for the multi time-step reconstruction is frozen flow. The results in the analytical evaluation, the E2E simulation, and the laboratory experiment with RAVEN are computed with the perfect frozen flow condition. Although we refer to the decay ratio of the temporal correlation of measurements from SH-WFSs as an indicator whether the frozen flow assumption is valid, it is still not clear how the boiling of the turbulence affects the performance of the multi time-step reconstruction. In order to understand the effect of the boiling 
of the turbulence and the limitation of the time difference of the previous time-steps, on-sky experiments are needed. The computational complexity of the multi time-step reconstructor for ELT-sized systems is one of the points that must be considered. For instance, GPGPU can be used for accelerating the computation, and the predictive control can relax the temporal constraints.

\section{APPENDIX A: ANALYTICAL TOMOGRAPHIC ERROR}

The tip-tilt removed tomographic error in the direction $\boldsymbol{\theta}_{\boldsymbol{k}}$, $\sigma_{\text {tomo }}^{2}\left(\boldsymbol{\theta}_{k}\right)$, is defined as

$$
\sigma_{\text {tomo }}^{2}\left(\boldsymbol{\theta}_{\boldsymbol{k}}\right)=\left\langle\left\|\boldsymbol{\varphi}_{\boldsymbol{k}}-\hat{\boldsymbol{\varphi}}_{\boldsymbol{k}}\right\|^{2}\right\rangle / n_{\text {node }},
$$

where $n_{\text {node }}$ is the number of valid nodes on the aperture plane, $\varphi_{k}$ and $\hat{\boldsymbol{\varphi}}_{k}$ are an actual and a reconstructed aperture-plane phase coming from the direction $\boldsymbol{\theta}_{\boldsymbol{k}}$, respectively, and \langle\rangle indicates an ensemble average over time. With Eq. (1), the actual phase is written as $\boldsymbol{\varphi}_{\boldsymbol{k}}=\boldsymbol{P}_{\boldsymbol{\theta}_{\boldsymbol{k}}} \boldsymbol{\phi}$. The reconstructed phase is expressed using Eqs. (5) and (6) as $\hat{\boldsymbol{\varphi}}_{\boldsymbol{k}}=\boldsymbol{P}_{\boldsymbol{\theta}_{\boldsymbol{k}}} \boldsymbol{E} \boldsymbol{\Gamma} \boldsymbol{P}_{g s} \boldsymbol{\phi}+\boldsymbol{P}_{\boldsymbol{\theta}_{\boldsymbol{k}}} \boldsymbol{E} \boldsymbol{\eta}$, where $\boldsymbol{E}$ is a reconstructor reconstructing the phase distortion due to each atmospheric turbulence layer. Using these expressions, Eq. (A1) is represented as

$$
\sigma_{\text {tomo }}^{2}\left(\boldsymbol{\theta}_{\boldsymbol{k}}\right)=\left\langle\left\|\boldsymbol{P}_{\boldsymbol{\theta}_{\boldsymbol{k}}}\left(\boldsymbol{I}-\boldsymbol{E} \boldsymbol{\Gamma} \boldsymbol{P}_{g_{s}}\right) \boldsymbol{\phi}-\boldsymbol{P}_{\boldsymbol{\theta}_{\boldsymbol{k}}} \boldsymbol{E} \boldsymbol{\eta}\right\|^{2}\right\rangle / n_{\text {node }}
$$

Since a variance of $\boldsymbol{x}$ can be rewritten with using a trace of a matrix as $\left\langle\|\boldsymbol{x}\|^{2}\right\rangle=\operatorname{Tr}\left[\left\langle\boldsymbol{x} \boldsymbol{x}^{T}\right\rangle\right]$, we can get Eq. (11), where we define that $\Sigma_{\boldsymbol{\phi}}=\left\langle\boldsymbol{\phi} \boldsymbol{\phi}^{T}\right\rangle, \Sigma_{\eta}=\left\langle\boldsymbol{\eta} \boldsymbol{\eta}^{T}\right\rangle$, and it is assumed that the phase distortion $\boldsymbol{\phi}$ is independent from the measurement noise $\boldsymbol{\eta},\left\langle\boldsymbol{\phi} \boldsymbol{\eta}^{T}\right\rangle=\left\langle\boldsymbol{\phi} \boldsymbol{\eta}^{T}\right\rangle=\mathbf{0}$.

Funding. Japan Society for the Promotion of Science (JSPS) (15J02510, 23740140, 26287027); Agence Nationale de la Recherche (ANR) (ANR-11-IDEX-0001-02.

Acknowledgment. Thanks to Naruhisha Takato, Yutaka Hayano, and Jean-Pierre Véran for many discussions. Thanks to the staff members of Subaru Telescope for their support. Y. Ono is supported by Japan Society for the Promotion of Science (JSPS) Grant-in-Aid for JSPS Fellows (15J02510). M. Akiyama is supported by JSPS Grant-in-Aid for Young Scientist (B) (23740140) and Grant-in-Aid for Scientific Research (B) (26287027). C. Correia is supported by Agence Nationale de la Recherche (ANR) (ANR-11-IDEX0001-02).

\section{REFERENCES}

1. Y. Hayano, H. Takami, S. Oya, M. Hattori, Y. Saito, M. Watanabe, O. Guyon, Y. Minowa, S. E. Egner, M. Ito, V. Garrel, S. Colley, T. Golota, and M. Iye, "Commissioning status of Subaru laser guide star adaptive optics system," Proc. SPIE 7736, 77360N (2010).

2. P. L. Wizinowich, D. L. Mignant, A. H. Boucheza, R. D. Campbell, J. C. Y. Chin, A. R. Contos, M. A. van Dam, S. K. Hartman, E. M. Johansson, R. E. Lafon, H. Lewis, P. J. Stomski, D. M. Summers, C. G. Brown, P. M. Danforth, C. E. Max, and D. M. Pennington, "The W. M. Keck Observatory laser guide star adaptive optics system: overview," Publ. Astron. Soc. Pac. 118, 297-309 (2006).

3. O. Lai, J.-P. Véran, G. Herriot, J. White, J. Ball, and C. Trujillo, "Altair performance and upgrades," Proc. SPIE 9148, 914838 (2014).
4. W. Skidmore, S. Els, T. Travouillon, R. Riddle, M. Schöck, E. Bustos, J. Seguel, and D. Walker, "Thirty Meter Telescope site testing V: seeing and isoplanatic angle," Publ. Astron. Soc. Pac. 121, 1151-1166 (2009).

5. J. Beckers, "Increasing the size of the anisoplanatic patch with multiconjugate adaptive optics," in Proceedings of ESO Conference (1988), Vol. 4007, pp. 693-703.

6. F. Hammer, F. Sayède, E. Gendron, T. Fusco, D. Burgarella, V. Cayatte, J. Conan, F. Courbin, H. Flores, I. Guinouard, L. Jocou, A. Lançon, G. Monnet, M. Mouhcine, F. Rigaud, D. Rouan, G. Rousset, V. Buat, and F. Zamkotsian, "The FALCON concept: multi-object spectroscopy combined with MCAO in near-IR," in Proceedings of ESO Workshop (2002), p. 139.

7. D. R. Andersen, S. S. Eikenberry, M. Fletcher, W. Gardhouse, B. Leckie, J.-P. Véran, D. Gavel, R. Clare, R. Guzman, L. Jolissaint, R. Julian, and W. Rambold, "The MOAO system of the IRMOS near-infrared multi-object spectrograph for TMT," Proc. SPIE 6269, 62694K (2006).

8. F. Hammer, B. Barbuy, J. G. Cuby, L. Kaper, S. Morris, C. J. Evans, P. Jagourel, G. Dalton, P. Rees, M. Puech, M. Rodrigues, D. Pearson, and K. Disseau, "MOSAIC at the E-ELT: a multi-object spectrograph for astrophysics, IGM and cosmology," Proc. SPIE 9147, 914727 (2014).

9. M. Akiyama, S. Oya, Y. H. Ono, H. Takami, S. Ozaki, Y. Hayano, I. Iwata, K. Hane, T. Wu, T. Yamamuro, and Y. Ikeda, "TMT-age: wide field of regard multi-object adaptive optics for TMT," Proc. SPIE 9148, 914814 (2014).

10. C. Correia, K. Jackson, J.-P. Véran, D. Andersen, O. Lardière, and C. Bradley, "Static and predictive tomographic reconstruction for wide-field multi-object adaptive optics systems," J. Opt. Soc. Am. A 31, 101-113 (2014).

11. C. M. Correia, K. Jackson, J.-P. Véran, D. Andersen, O. Lardière, and C. Bradley, "Spatio-angular minimum-variance tomographic controller for multi-object adaptive-optics systems," Appl. Opt. 54, 5281-5290 (2015).

12. S. M. Ammons, L. Poyneer, D. T. Gavel, R. Kupke, C. E. Max, and L. Johnson, "Evidence that wind prediction with multiple guide stars reduces tomographic errors and expands MOAO field of regard," Proc. SPIE 8447, 84471U (2012).

13. L. Poyneer, M. van Dam, and J.-P. Véran, "Experimental verification of the frozen flow atmospheric turbulence assumption with use of astronomical adaptive optics telemetry," J. Opt. Soc. Am. A 26, 833-846 (2009).

14. A. Guesalaga, B. Neichel, A. Cortés, C. Béchet, and D. Guzmán, "Using the $C_{n}^{2}$ and wind profiler method with wide-field laser-guidestars adaptive optics to quantify the frozen-flow decay," Mon. Not. R. Astron. Soc. 440, 1925-1933 (2014).

15. O. Lardière, D. Andersen, C. Blain, C. Bradley, D. Gamroth, K. Jackson, P. Lach, R. Nash, K. Venn, J.-P. Véran, C. Correia, S. Oya, Y. Hayano, H. Terada, Y. Ono, and M. Akiyama, "Multi-object adaptive optics on-sky results with Raven," Proc. SPIE 9148, 91481G (2014).

16. B. L. Ellerbroek, "Efficient computation of minimum-variance wavefront reconstructors with sparse matrix techniques," J. Opt. Soc. Am. A 19, 1803-1816 (2002).

17. L. Gilles, P. Massioni, C. Kulcsár, H.-F. Raynaud, and B. Ellerbroek, "Distributed Kalman filtering compared to Fourier domain preconditioned conjugate gradient for laser guide star tomography on extremely large telescopes," J. Opt. Soc. Am. A 30, 898-909 (2013).

18. L. H. Lee, "Sparse-matrix regularization for minimum-variance reconstruction of pseudo-Kolmogorov turbulence," in Adaptive Optics: Analysis and Methods/Computational Optical Sensing and Imaging/Information Photonics/Signal Recovery and Synthesis Topical Meetings on CD-ROM (Optical Society of America, 2007), paper JTuA2.

19. M. Schöck and E. J. Spillar, "Method for a quantitative investigation of the frozen flow hypothesis," J. Opt. Soc. Am. A 17, 1650-1658 (2000).

20. L. Gilles, C. R. Vogel, and B. L. Ellerbroek, "Multigrid preconditioned conjugate-gradient method for large-scale wave-front reconstruction," J. Opt. Soc. Am. A 19, 1817-1822 (2002).

21. Q. Yang, C. R. Vogel, and B. L. Ellerbroek, "Fourier domain preconditioned conjugate gradient algorithm for atmospheric tomography," Appl. Opt. 45, 5281-5293 (2006). 
22. L. Lessard, M. West, D. MacMynowski, and S. Lall, "Warm-started wavefront reconstruction for adaptive optics," J. Opt. Soc. Am. A 25, 1147-1155 (2008).

23. L. Gilles and B. Ellerbroek, "Shack-Hartmann wavefront sensing with elongated sodium laser beacons: centroiding versus matched filtering," Appl. Opt. 45, 6568-6576 (2006).

24. D. R. Andersen, K. J. Jackson, C. Blain, C. Bradley, C. Correia, M. Ito, O. Lardière, and J.-P. Véran, "Performance modeling for the RAVEN multi-object adaptive optics demonstrator," Publ. Astron. Soc. Pac. 124, 469-484 (2012).

25. A. Miyashita, N. Takato, T. Usuda, F. Uraguchi, and R. Ogasawara, "Statistics of the weather data, environment data, and the seeing of the Subaru Telescope," Proc. SPIE 5489, 207-217 (2004).

26. S. G. Els, T. Travouillon, M. Schöck, R. Riddle, W. Skidmore, J. Seguel, E. Bustos, and D. Walker, "Thirty Meter Telescope site testing VI: turbulence profiles," Publ. Astron. Soc. Pac. 121, pp. 527-543 (2009).

27. J. W. Hardy, Adoptive Optics for Astronomical Telescopes (Oxford University, 1998).
28. R. W. Wilson, "SLODAR: measuring optical turbulence altitude with a Shack-Hartmann wavefront sensor," Mon. Not. R. Astron. Soc. 337 103-108 (2002).

29. T. Butterley, R. W. Wilson, and M. Sarazin, "Determination of the profile of atmospheric optical turbulence strength from SLODAR data," Mon. Not. R. Astron. Soc. 369, 835-845 (2006).

30. A. Cortés, B. Neichel, A. Guesalaga, J. Osborn, F. Rigaut, and D. Guzman, "Atmospheric turbulence profiling using multiple laser star wavefront sensors," Mon. Not. R. Astron. Soc. 427, 2089-2099 (2012).

31. J.-F. Lavigne, F. Lamontagne, G. Anctil, M. Wang, M. Tremblay, O. Lardière, R. Nash, D. Andersen, M. Savard, P. Côté, C. H. Bradley, and F. Châteauneuf, "Design and test results of the calibration unit for the MOAO demonstrator RAVEN," Proc. SPIE 8447, 844754 (2012).

32. Y. H. Ono, M. Akiyama, and S. Oya, "TMT-AGE: numerical simulation of a new tomographic reconstruction method for wide for MOAO," Proc. SPIE 9148, 91486M (2014). 